\title{
Spectral gap for the Cauchy process on convex, symmetric domains
}

\author{
Rodrigo Bañuelos* \\ Department of Mathematics \\ Purdue University \\ West Lafayette, IN 47906 \\ banuelos@math.purdue.edu
}

\author{
Tadeusz Kulczycki ${ }^{\dagger}$ \\ Institute of Mathematics \\ Wrocław University of Technology \\ 50-370 Wrocław, Poland \\ tkulczyc@im.pwr.wroc.pl
}

August 28, 2005

\begin{abstract}
Let $D \subset \mathbb{R}^{2}$ be a bounded convex domain which is symmetric relative to both coordinate axes. Assume that $[-a, a] \times[-b, b], a \geq$ $b>0$ is the smallest rectangle (with sides parallel to the coordinate axes) containing $D$. Let $\left\{\lambda_{n}\right\}_{n=1}^{\infty}$ be the eigenvalues corresponding to the semigroup of the Cauchy process killed upon exiting $D$. We obtain the following estimate on the spectral gap:

$$
\lambda_{2}-\lambda_{1} \geq \frac{C b}{a^{2}}
$$

where $C$ is an absolute constant. The estimate is obtained by proving new weighted Poincaré inequalities and appealing to the connection between the eigenvalue problem for the Cauchy process and a mixed boundary value problem for the Laplacian in one dimension higher known as the mixed Steklov problem established in [5].
\end{abstract}

\section{Contents}

$\S 1$. Introduction and Statement of Results

$\S 2$. Geometric and Analytic Properties of $\varphi_{1}$

$\S 3$. Weighted Poincaré inequalities

$\S 4$. The Spectral Gap Estimate, Proof of Theorem 1.1

${ }^{*}$ Supported in part by NSF Grant \# 9700585-DMS

${ }^{\dagger}$ Supported by KBN grant 1 P03A 02028 and RTN Harmonic Analysis and Related Problems, contract HPRN-CT-2001-00273-HARP 


\section{Introduction and Statement of Results}

Spectral gap estimates for eigenvalues of the Laplacian with Dirichlet boundary conditions, henceforth referred to as the Dirichlet Laplacian, have attracted considerable attention for many years as evident by the many papers written on this subject. See for example, [1], [2], [3], [10], [28], [29], [32], [31], [34]. The Dirichlet Laplacian is the infinitesimal generator of the semigroup of Brownian motion killed upon leaving a domain. Therefore questions concerning eigenvalues of this operator have been studied both by analytic and probabilistic methods. The question of precise lower bounds for the spectral gap for the Dirichlet Laplacian (the difference between the first two eigenvalues) and for Schrödinger operators with non-negative convex potentials, was raised by M. van den Berg [10] (see also [1], [2], and problem \#44 in [33]). The question was motivated by problems in mathematical physics related to the behavior of free Boson gases. The van den Berg conjecture asserts that for any convex bounded domain of diameter $d$, the spectral gap is bounded below by $3 \pi^{2} / d^{2}$ and that this should hold not only for the Dirichlet Laplacian but also for Schrödinger operators with convex non-negative potentials. The full conjecture is only known in dimension one, see [28]. For zero potentials it has been proved in [9] and [20] for certain planar domains with symmetry. From the probabilistic point of view, the spectral gap determines the rate to equilibrium for Brownian motion conditioned to remain forever in $D$, the Doob $h$-process corresponding to the ground state eigenfunction.

The natural question arises as to whether spectral gap bounds for the Laplacian, such as those discussed above, can be extended to non-local, pseudo-differential operators. The class of such operators which are most closely related to the Laplacian $\Delta$ from the point of view of Brownian motion are $-(-\Delta)^{\alpha / 2}, \alpha \in(0,2)$. These are the infinitesimal generators of the symmetric Lévy $\alpha$-stable processes. These processes do not have continuous paths; a fact related to the non-locality of the operator $-(-\Delta)^{\alpha / 2}$. As in the case of Brownian motion, nevertheless, we can consider the semigroup of these processes killed upon exiting domains and we can consider the eigenvalues of such semigroups. Here again, the spectral gap determines the asymptotic exponential rate of convergence to equilibrium for the process conditioned to remain forever in the domain. By abuse of language, instead of speaking of the eigenvalue gap for the operator $-(-\Delta)^{\alpha / 2}$ we will often refer to it as the eigenvalue gap for the corresponding process.

The purpose of this paper is to obtain spectral gap estimates for the Cauchy process. This is the symmetric $\alpha$-stable process of order $\alpha=1$

corresponding to the square root of the Laplacian. We do this by by proving 
new weighted Poincaré inequalities and exploring the connection between the eigenvalue problem for the Cauchy process and the mixed Steklov problem established in [5]. These results raise many natural questions concerning spectral gap bounds for other symmetric $\alpha$-stable processes and for more general Lévy processes. We believe that as with the results in [5] which have already motivated subsequent work by many others (see [21], [22] [18]), the current results will also be of interest and will open doors to further explorations.

Before we state our results we make some of the above notions more precise and introduce some notation. Let $X_{t}$ be a symmetric $\alpha$-stable process in $\mathbb{R}^{d}, \alpha \in(0,2]$. This is a process with independent and stationary increments and characteristic function $E^{0} e^{i \xi X_{t}}=e^{-t|\xi|^{\alpha}}, \xi \in \mathbb{R}^{d}, t>0$. We will use $E_{x}, P_{x}$ to denote the expectation and probability of this process starting at $x$, respectively. By $p^{(\alpha)}(t, x, y)=p_{t}^{(\alpha)}(x-y)$ we will denote the transition density of this process. That is,

$$
P_{x}\left(X_{t} \in B\right)=\int_{B} p^{(\alpha)}(t, x, y) d y .
$$

When $\alpha=2$ the process $X_{t}$ is just the Brownian motion in $\mathbb{R}^{d}$ running at twice the speed. That is, if $\alpha=2$ then

$$
p^{(2)}(t, x, y)=\frac{1}{(4 \pi t)^{d / 2}} e^{\frac{-|x-y|^{2}}{4 t}}, \quad t>0, x, y \in \mathbb{R}^{d} .
$$

When $\alpha=1$, the process $X_{t}$ is the Cauchy process in $\mathbb{R}^{d}$ whose transition densities are given by

$$
p^{(1)}(t, x, y)=\frac{c_{d} t}{\left(t^{2}+|x-y|^{2}\right)^{(d+1) / 2}}, \quad t>0, x, y \in \mathbb{R}^{d}
$$

where

$$
c_{d}=\Gamma((d+1) / 2) / \pi^{(d+1) / 2} .
$$

Our main concern in this paper are the eigenvalues of the semigroup of the process $X_{t}$ killed upon leaving a domain. Let $D \subset \mathbb{R}^{d}$ be a bounded connected domain and $\tau_{D}=\inf \left\{t \geq 0: X_{t} \notin D\right\}$ be the first exit time of $D$. By $\left\{T_{t}^{D}\right\}_{t \geq 0}$ we denote the semigroup on $L^{2}(D)$ of $X_{t}$ killed upon exiting $D$. That is,

$$
T_{t}^{D} f(x)=E_{x}\left(f\left(X_{t}\right), \tau_{D}>t\right), \quad x \in D, t>0, f \in L^{2}(D) .
$$


The semigroup has transition densities $p_{D}(t, x, y)$ satisfying

$$
T_{t}^{D} f(x)=\int_{D} p_{D}(t, x, y) f(y) d y
$$

The kernel $p_{D}(t, x, y)$ is strictly positive symmetric and

$$
p_{D}(t, x, y) \leq p^{(\alpha)}(t, x, y) \leq c_{\alpha, d} t^{-d / \alpha}, \quad x, y \in D, t>0 .
$$

The fact that $D$ is bounded implies that for any $t>0$ the operator $T_{t}^{D}$ maps $L^{2}(D)$ into $L^{\infty}(D)$. From the general theory of semigroups (see [19]) it follows that there exists an orthonormal basis of eigenfunctions $\left\{\varphi_{n}\right\}_{n=1}^{\infty}$ for $L^{2}(D)$ and corresponding eigenvalues $\left\{\lambda_{n}\right\}_{n=1}^{\infty}$ satisfying

$$
0<\lambda_{1}<\lambda_{2} \leq \lambda_{3} \leq \ldots
$$

with $\lambda_{n} \rightarrow \infty$ as $n \rightarrow \infty$. That is, the pair $\left\{\varphi_{n}, \lambda_{n}\right\}$ satisfies

$$
T_{t}^{D} \varphi_{n}(x)=e^{-\lambda_{n} t} \varphi_{n}(x), \quad x \in D, t>0 .
$$

The eigenfunctions $\varphi_{n}$ are continuous and bounded on $D$. In addition, $\lambda_{1}$ is simple and the corresponding eigenfunction $\varphi_{1}$, often called the ground state eigenfunction, is strictly positive on $D$. For more general properties of the semigroups $\left\{T_{t}^{D}\right\}_{t \geq 0}$, see [24], [12], [16].

It is well known (see [4], [16], [17], [27]) that if $D$ is a bounded connected Lipschitz domain and $\alpha=2$, or that if $D$ is a bounded connected domain for $0<\alpha<2$, then $\left\{T_{t}^{D}\right\}_{t \geq 0}$ is intrinsically ultracontractive. Intrinsic ultracontractivity is a remarkable property with many consequences. It implies, in particular, that

$$
\lim _{t \rightarrow \infty} \frac{e^{\lambda_{1} t} p_{D}(t, x, y)}{\varphi_{1}(x) \varphi_{1}(y)}=1,
$$

uniformly in both variables $x, y \in D$. In addition, the rate of convergence is given by the spectral gap $\lambda_{2}-\lambda_{1}$. That is, for any $t \geq 1$ we have

$$
e^{-\left(\lambda_{2}-\lambda_{1}\right) t} \leq \sup _{x, y \in D}\left|\frac{e^{\lambda_{1} t} p_{D}(t, x, y)}{\varphi_{1}(x) \varphi_{1}(y)}-1\right| \leq C(D, \alpha) e^{-\left(\lambda_{2}-\lambda_{1}\right) t} .
$$

The proof of this for $\alpha=2$ may be found in [32]. The proof in our setting is exactly the same.

In the Brownian motion case the properties of eigenfunctions and eigenvalues have been extensively studied for many years, both analytically and 
probabilistically. It is well known that geometric information on $D$, such as convexity, symmetry, volume growth, smoothness of its boundary, etc., provide information not only on the ground state eigenfunction $\varphi_{1}$ and the ground state eigenvalue $\lambda_{1}$, but also on the spectral gap $\lambda_{2}-\lambda_{1}$, and on the geometry of the nodal domains of $\varphi_{2}$.

In the case of stable processes of index $0<\alpha<2$ surprisingly very little seems to be known. We refer the reader to [6] where some of the known results are reviewed and for a discussion of the many questions that remain open. In the case of the one dimensional interval the spectral gap for the Cauchy process has been estimated in [5] (see Theorem 5.3 and Corollary 2.2). Recently, Z.-Q. Chen and R. Song in [18] (see (5.1)) obtained some estimates of eigenvalues for all $\alpha$-stable symmetric processes which can be used to obtain estimates of the spectral gap for $\alpha$-stable processes in dimension one, when $\alpha>1$. However, except for the one-dimensional case, lower bound estimates on $\lambda_{2}-\lambda_{1}$ remain completely open. In [6], we studied domains with one axis of symmetry and obtained estimates for $\lambda_{*}-\lambda_{1}$ in the Cauchy process case where $\lambda_{*}$ is the eigenvalue corresponding to the "first" antisymmetric eigenfunction for $D$. However, due to the fact that we do not know the location of the nodal lines for the second eigenfunction for symmetric domains (an interesting question on its own right), the results from [6] do not give estimates on the spectral gap $\lambda_{2}-\lambda_{1}$. In this paper we restrict ourselves to bounded convex planar domains which are symmetric with respect to both coordinate axes. For these domains we are able to obtain estimates on the spectral gap for the Cauchy process. Here is the main result of this paper.

Theorem 1.1. Let $D \subset \mathbb{R}^{2}$ be a bounded convex domain which is symmetric relative to both coordinate axes. Assume that $[-L, L] \times[-1,1], L \geq 1$, is the smallest rectangle (with sides parallel to the coordinate axes) containing $D$. Let $\left\{T_{t}^{D}\right\}_{t \geq 0}$ be the semigroup of the Cauchy process killed upon exiting $D$ with eigenvalues $\left\{\lambda_{n}\right\}_{n=1}^{\infty}$. Then we have

$$
\lambda_{2}-\lambda_{1} \geq \frac{C}{L^{2}},
$$

where $C=10^{-7}$ is an absolute constant.

The eigenvalues $\lambda_{n}$ satisfies the scaling property $\lambda_{n}(k D)=\lambda_{n}(D) / k$, $k>0$. This leads to the following easy corollary.

Corollary 1.1. Let $D \subset \mathbb{R}^{2}$ satisfy the assumptions of Theorem 1.1 except that now the smallest rectangle containing $D$ is $[-a, a] \times[-b, b], a \geq b>0$. 
Then we have

$$
\lambda_{2}-\lambda_{1} \geq \frac{C b}{a^{2}},
$$

where $C$ is the same constant as in Theorem 1.1.

Remark 1.1. We remark here that for any bounded convex domain in $\mathbb{R}^{d}$, $d \geq 2$, of inradius $r_{D}$ (the radius of the largest ball contained in $D$ ), we have

$$
\lambda_{2}-\lambda_{1} \leq \frac{\sqrt{\mu_{2}}-\frac{1}{2} \sqrt{\mu_{1}}}{r_{D}}
$$

where $\mu_{1}$ and $\mu_{2}$ are, respectively, the first and second eigenvalues for the Laplacian for the unit ball, $B(0,1)$, in $\mathbb{R}^{d}$. In fact, $\mu_{2}=j_{d / 2,1}^{2}$ and $\mu_{1}=$ $j_{d / 2-1,1}^{2}$ where $j_{p, k}$ denotes the $k^{t h}$ positive zero of the Bessel function $J_{p}(x)$. This estimate, and its version for all $\alpha \in(0,2)$, is proved in [6]. From this point on we will concentrate ourselves on the lower bound for the gap and upper bounds will not be mentioned any further. (We refer the reader to [6] for further questions related to the upper bound including a version of the Payne-Pólya-Weinberger Conjecture for stable processes.)

\section{Geometric and Analytic Properties of $\varphi_{1}$}

We start with the following theorem which as its proof shows, holds for any stable processes of order $0<\alpha \leq 2$ and other Lévy processes obtained from Brownian motion by subordination.

Theorem 2.1. Let $D \subset \mathbb{R}^{2}$ satisfy the assumptions of Theorem 1.1. Let $\varphi_{1}$ be the first eigenfunction of the Cauchy semigroup $\left\{T_{t}^{D}\right\}_{t \geq 0}$. Then we have

(i) $\varphi_{1}$ is continuous and strictly positive in $D$.

(ii) $\varphi_{1}$ is symmetric in $D$ with respect to both coordinate axes. That is, $\varphi_{1}\left(x_{1},-x_{2}\right)=\varphi_{1}\left(x_{1}, x_{2}\right)$ and $\varphi_{1}\left(-x_{1}, x_{2}\right)=\varphi_{1}\left(x_{1}, x_{2}\right)$.

(iii) $\varphi_{1}$ is unimodal in $D$ with respect to both coordinate axes. That is, if we take any $a_{2} \in(-1,1)$ and $p\left(a_{2}\right)>0$ such that $\left(p\left(a_{2}\right), a_{2}\right) \in \partial D$, then the function $v\left(x_{1}\right)=\varphi_{1}\left(x_{1}, a_{2}\right)$ defined on $\left(-p\left(a_{2}\right), p\left(a_{2}\right)\right)$ is nondecreasing on $\left(-p\left(a_{2}\right), 0\right)$ and non-increasing on $\left(0, p\left(a_{2}\right)\right)$. Similarly, if we take any $a_{1} \in(-L, L)$ and $r\left(a_{1}\right)>0$ such that $\left(a_{1}, r\left(a_{1}\right)\right) \in \partial D$, then the function $u\left(x_{2}\right)=\varphi_{1}\left(a_{1}, x_{2}\right)$ defined on $\left(-r\left(a_{1}\right), r\left(a_{1}\right)\right)$ is nondecreasing on $\left(-r\left(a_{1}\right), 0\right)$ and non-increasing on $\left(0, r\left(a_{1}\right)\right)$. 
The fact that $\varphi_{1}$ is continuous has already been proved in [5]. In fact, as shown there (Theorem 4.1) the eigenfunctions $\varphi_{n}$ are real analytic in $D$. This is true not only for the Cauchy process but also for all symmetric stable processes of order $\alpha \in(0,2)$. Also, the fact that $\varphi_{1}$ is strictly positive is well known. It follows from the general theory of semigroups and from the Harnack inequality proved below. Thus we only need to prove (ii) and (iii) above. These will follow from subordination and the following lemma.

Lemma 2.2. Let $B_{t}$ be Brownian motion in $\mathbb{R}^{2}$ and let $D \subset \mathbb{R}^{2}$ satisfy the assumptions of Theorem 1.1. For $x=\left(x_{1}, x_{2}\right) \in D$ and $0<t_{1}<t_{2}<\cdots<$ $t_{n}<\infty$, set

$$
F(x)=P_{x}\left\{B_{t_{1}} \in D, B_{t_{2}} \in D, \ldots, B_{t_{n}} \in D\right\}
$$

Then properties (ii) and (iii) of Theorem 2.1 hold for $F$. That is, F is symmetric and unimodal in $D$ with respect to both coordinate axes.

Let us assume the lemma for the moment. We recall that for $0<\alpha<2$ the symmetric stable process $X_{t}$ in $\mathbb{R}^{d}$ has the representation

$$
X_{t}=B_{2 \sigma_{t}},
$$

where $\sigma_{t}$ is a stable subordinator of index $\alpha / 2$ independent of $B_{t}$ (see [11]). Thus

$$
p_{t}^{(\alpha)}(x-y)=\int_{0}^{\infty} p_{s}^{(2)}(x-y) g_{\alpha / 2}(t, s) d s
$$

where $g_{\alpha / 2}(t, s)$ is the transition density of $\sigma_{t}$ and $p_{s}^{(2)}(x-y)$ is the Gaussian density with $t$ replaced by $2 t$ as in (1.1). Now, let $D$ and $t_{1}, t_{2}, \ldots, t_{n}$ be as in the statement of Lemma 2.2. Set $z_{0}=x$ and $t_{0}=0$. Using the Markov property of the stable process $X_{t}$, the subordination formula (2.2), Fubini's theorem, and the Markov property of the Brownian motion, in this order, 
we obtain that,

$$
\begin{aligned}
H(x)= & P_{x}\left\{X_{t_{1}} \in D, \ldots, X_{t_{n}} \in D\right\} \\
= & \int_{D} \cdots \int_{D} \prod_{i=1}^{n} p_{t_{i}-t_{i-1}}^{(\alpha)}\left(z_{i-1}-z_{i}\right) d z_{1} \ldots d z_{n} \\
= & \int_{0}^{\infty} \cdots \int_{0}^{\infty}\left(\int_{D} \cdots \int_{D} \prod_{i=1}^{n} p_{s_{i}}^{(2)}\left(z_{i-1}-z_{i}\right) d z_{1} \ldots d z_{n}\right) \\
& \times \prod_{i=1}^{n} g_{\alpha / 2}\left(t_{i}-t_{i-1}, s_{i}\right) d s_{1} \ldots d s_{n} \\
= & \int_{0}^{\infty} \ldots \int_{0}^{\infty} P_{x}\left\{B_{2 s_{1}} \in D, B_{2\left(s_{1}+s_{2}\right)} \in D, \ldots, B_{2\left(s_{1}+s_{2}+\cdots+s_{n}\right)} \in D\right\} \\
& \times \prod_{i=1}^{n} g_{\alpha / 2}\left(t_{i}-t_{i-1}, s_{i}\right) d s_{1} \ldots d s_{n} .
\end{aligned}
$$

From this and Lemma 2.2 we see that the function

$$
H(x)=P_{x}\left\{X_{t_{1}} \in D, \ldots, X_{t_{n}} \in D\right\}
$$

also satisfies the assertions (ii) and (iii) of Theorem 2.1.

Let us now denote by $\tau_{D}^{\alpha}$ the first exit time of the symmetric stable process from $D . D$ is intrinsically ultracontractive (see [27]) and we have that

$$
\varphi_{1}^{\alpha}(x)=c(\alpha) \lim _{t \rightarrow \infty} e^{\lambda_{1}^{\alpha} t} P_{x}\left\{\tau_{D}^{\alpha}>t\right\},
$$

where $\lambda_{1}^{\alpha}$ is the first eigenvalue for the semigroup of the stable process killed upon leaving $D$ and $\varphi_{1}^{\alpha}(x)$ is its corresponding eigenfunction. Furthermore, the convergence in (2.3) is uniform for $x \in D$. Thus to prove properties (ii) and (iii) of Theorem 2.1 for $\varphi_{1}^{\alpha}(x)$, it is enough to prove them for $P_{x}\left\{\tau_{D}^{\alpha}>t\right\}$. By the right continuity of the sample paths and the fact that for our domains $P_{x}\left\{X_{\tau_{D}^{\alpha}} \in \partial D\right\}=0$ for $x \in D$ we have

$$
\begin{aligned}
P_{x}\left\{\tau_{D}^{\alpha}>t\right\} & =P_{z}\left\{X_{s} \in D, 0 \leq s \leq t\right\} \\
& =\lim _{n \rightarrow \infty} P_{x}\left\{X_{\frac{i t}{n}} \in D, i=1, \ldots, n\right\} .
\end{aligned}
$$

Thus $P_{x}\left\{\tau_{D}^{\alpha}>t\right\}$ satisfies properties (ii) and (iii) of Theorem 2.1 and hence so does $\varphi_{1}^{\alpha}(x)$. 
Proof of Lemma 2.2. By the symmetry properties of $D$ and the the reflection properties of Brownian motion, $F$ is symmetric relative to both coordinate axes. That is, $F$ satisfies (ii) of Theorem 2.1. With $z_{0}=x=\left(x_{1}, x_{2}\right)$ and $t_{0}=0$ we have as above that

$$
F(x)=\int_{D} \ldots \int_{D} \prod_{i=1}^{n} p_{t_{i}-t_{i-1}}^{\mathbb{R}^{2}}\left(z_{i-1}-z_{i}\right) d z_{1} \ldots d z_{n},
$$

where

$$
p_{t}^{\mathbb{R}^{2}}(z, w)=\frac{1}{2 \pi t} e^{-\frac{|z-w|^{2}}{2 t}}
$$

is the Gaussian density in $\mathbb{R}^{2}$. Setting $z=\left(u_{1}, v_{1}\right)$ and $w=\left(u_{2}, v_{2}\right)$ we see that

$$
p_{t}^{\mathbb{R}^{2}}(z-w)=p_{t}^{\mathbb{R}}\left(u_{1}-u_{2}\right) p_{t}^{\mathbb{R}}\left(v_{1}-v_{2}\right),
$$

where

$$
p_{t}^{\mathbb{R}}(r)=\frac{1}{\sqrt{2 \pi t}} e^{-\frac{r^{2}}{2 t}}
$$

is the standard one dimensional Gaussian distribution. Let us now write the domain as $D=\left\{(u, v) \in \mathbf{R}^{2}: v \in(-1,1),-f(v)<u<f(v)\right\}$ for some function $f$ so that $L=\sup _{(u, 0) \in D}|u|$. With $t_{0}=0$ and $x=\left(x_{1}, x_{2}\right)=$ $\left(u_{0}, v_{0}\right),(2.5)$ gives

$$
F(x)=\int_{-1}^{1} \cdots \int_{-1}^{1} \prod_{i=1}^{n} p_{t_{i}-t_{i-1}}^{\mathbb{R}}\left(v_{i}-v_{i-1}\right) \Psi_{n}\left(x_{1} ; v_{1}, \ldots, v_{n}\right) d v_{1} \cdots d v_{n}
$$

where

$$
\Psi_{n}\left(x_{1} ; v_{1}, \ldots, v_{n}\right)=\int_{-f\left(v_{1}\right)}^{f\left(v_{1}\right)} \cdots \int_{-f\left(v_{n}\right)}^{f\left(v_{n}\right)} \prod_{i=1}^{n} p_{t_{i}-t_{i-1}}^{\mathbb{R}}\left(u_{i}-u_{i-1}\right) d u_{1} \cdots d u_{n}
$$

Fix $v_{1}, v_{2}, \ldots, v_{n}$ and set $a_{1}=f\left(v_{1}\right), \ldots, a_{n}=f\left(v_{n}\right)$. Let

$$
\Phi_{n}\left(x_{1}\right)=\int_{-a_{1}}^{a_{1}} \cdots \int_{-a_{n}}^{a_{n}} \prod_{i=1}^{n} p_{t_{i}-t_{i-1}}^{\mathbb{R}}\left(u_{i}-u_{i-1}\right) d u_{1} \cdots d u_{n}
$$

where as above $x_{1}=u_{0}$. Let us observe that

$$
\begin{aligned}
& \Phi_{n}\left(x_{1}\right)=\int_{-a_{1}}^{a_{1}} \cdots \int_{-a_{n}}^{a_{n}} p_{t_{1}}^{\mathbb{R}}\left(u_{1}-x_{1}\right) \prod_{i=2}^{n} p_{t_{i}-t_{i-1}}^{\mathbb{R}}\left(u_{i}-u_{i-1}\right) d u_{1} \ldots d u_{n} \\
= & \int_{-a_{1}}^{a_{1}} p_{t_{1}}^{\mathbb{R}}\left(u_{1}-x_{1}\right)\left(\int_{-a_{2}}^{a_{2}} \cdots \int_{-a_{n}}^{a_{n}} \prod_{i=2}^{n} p_{t_{i}-t_{i-1}}^{\mathbb{R}}\left(u_{i}-u_{i-1}\right) d u_{2} \ldots d u_{n}\right) d u_{1} \\
= & \int_{-a_{1}}^{a_{1}} p_{t_{1}}^{\mathbb{R}}\left(u_{1}-x_{1}\right) \Phi_{n-1}\left(u_{1}\right) d u_{1}
\end{aligned}
$$


where

$$
\Phi_{n-1}\left(u_{1}\right)=\int_{-a_{2}}^{a_{2}} \ldots \int_{-a_{n}}^{a_{n}} \prod_{i=2}^{n} p_{t_{i}-t_{i-1}}^{\mathbb{R}}\left(u_{i}-u_{i-1}\right) d u_{2} \ldots d u_{n} .
$$

With this, we have

$$
\Phi_{n}\left(x_{1}\right)=\frac{1}{\sqrt{2 \pi t_{1}}} \int_{-a_{1}}^{a_{1}} e^{-\frac{\left(u_{1}-x_{1}\right)^{2}}{2 t_{1}}} \Phi_{n-1}\left(u_{1}\right) d u_{1} .
$$

Using the same arguments as in the proof of Lemma 2.1 in [7] one can show that $\Phi_{n}\left(x_{1}\right)$ is decreasing on $(0, \infty)$ and increasing on $(-\infty, 0)$, for all $n \geq 1$. Thus it follows that $\frac{\partial F}{\partial x_{1}}\left(x_{1}, x_{2}\right) \geq 0$, for $x_{1}<0$ and $\frac{\partial F}{\partial x_{1}}\left(x_{1}, x_{2}\right) \leq 0$, for $x_{1}>0$. This completes the proof of Lemma 2.2.

Next, we prove the Harnack inequality. While this is well known for all symmetric stable processes, our purpose here is to give a proof in the case of the Cauchy process which will give an explicit (and reasonable) constant. The available proofs (see e.g. Theorem 6.1 in [14]) do not seem to provide such a constant.

Theorem 2.3. Let $D \subset \mathbb{R}^{2}$ satisfy the assumptions of Theorem 1.1. Let $\varphi_{1}$ be the first eigenfunction of the Cauchy semigroup $\left\{T_{t}^{D}\right\}_{t \geq 0}$. If $B\left(x_{0}, 3 / 8\right) \subset$ $D$ then on $B\left(x_{0}, 1 / 8\right) \varphi_{1}$ satisfies the Harnack inequality with the constant $C=70.5$. That is, for any $x, y \in B\left(x_{0}, 1 / 8\right)$ we have $\varphi_{1}(x) \leq C \varphi_{1}(y)$ where $C=70.5$.

Corollary 2.1. Let $D \subset \mathbb{R}^{2}$ satisfy the assumptions of Theorem 1.1. Let $\varphi_{1}$ be the first eigenfunction of the Cauchy semigroup $\left\{T_{t}^{D}\right\}_{t \geq 0}$. If $B\left(x_{0}, 3 / 8\right) \subset$ $D$ then on $B\left(x_{0}, 1 / 8\right) \varphi_{1}^{2}$ satisfies the Harnack inequality with the constant $C=5 \cdot 10^{3}$. That is, for any $x, y \in B\left(x_{0}, 1 / 8\right)$ we have $\varphi_{1}^{2}(x) \leq C \varphi_{1}^{2}(y)$ with $C=5 \cdot 10^{3}$

Proof of Theorem 2.3. Let $B \subset D$ be any ball $(B \neq D) . \quad G_{B}(x, y)=$ $\int_{0}^{\infty} p_{B}(t, x, y) d t$ is the Green function for $B$, where $p_{B}(t, x, y)$ is the density of the Cauchy process killed upon exiting $B, x, y \in B$. We have

$$
p_{B}(t, x, y)=\sum_{n=1}^{\infty} e^{-\lambda_{n}(B) t} \varphi_{n, B}(x) \varphi_{n, B}(y),
$$

where $\lambda_{n}(B)$ and $\varphi_{n, B}$ are the eigenvalues and eigenfunctions for the semi$\operatorname{group}\left\{T_{t}^{B}\right\}_{t \geq 0}$. 
We will use the fact that the first eigenfunction is $q$-harmonic in $B \subset D$ according to the 1-stable Schrödinger operator.

Let $\varphi_{1}, \lambda_{1}=\lambda_{1}(D)$ be the first eigenfunction and eigenvalue for the semigroup $\left\{T_{t}^{D}\right\}_{t \geq 0}$. Let $A$ be the infinitesimal generator of this semigroup. For $x \in D$ we have

$$
A \varphi_{1}(x)=\lim _{t \rightarrow 0^{+}} \frac{T_{t}^{D} \varphi_{1}-\varphi_{1}(x)}{t}=\frac{e^{-\lambda_{1}(D) t} \varphi_{1}(x)-\varphi_{1}(x)}{t}=-\lambda_{1}(D) \varphi_{1}(x) .
$$

This gives that $\left(A+\lambda_{1}(D)\right) \varphi_{1}=0$ on $D$. Let $B \subset D$ be any ball $(B \neq D)$. It follows that $\varphi_{1}$ is $q$-harmonic on $B$ according to the 1-stable Schrödinger operator $A+q$ with $q \equiv \lambda_{1}(D)$. Formally this follows from Proposition 3.17, Theorem 5.5, Definition 5.1 from [14] and the fact that $\left(B, \lambda_{1}(D)\right)$ is gaugable because $B$ is a proper open subset of $D$ and $\lambda_{1}(B)>\lambda_{1}(D)$.

Let $V_{B}(x, y)=\int_{0}^{\infty} e^{\lambda_{1}(D) t} p_{B}(t, x, y) d t$. Here, $V_{B}$ is the $q$-Green function, for $q \equiv \lambda_{1}(D)$, see page 58 in [14]. The $q$-harmonicity of $\varphi_{1}$ (Definition 5.1 in [14]), Theorem 4.10 in [14] (formula (4.15)) and formula (2.17) in [14] (page 61) give that for $z \in B$,

$$
\begin{aligned}
\varphi_{1}(z) & =E^{z}\left[e_{\lambda_{1}(D)}\left(\tau_{B}\right) \varphi_{1}\left(X\left(\tau_{B}\right)\right)\right] \\
& =\int_{B} V_{B}(z, y) \int_{D \backslash B}(2 \pi)^{-1}|y-w|^{-3} \varphi_{1}(w) d w d y
\end{aligned}
$$

where $e_{\lambda_{1}(D)}\left(\tau_{B}\right)=\exp \left(\lambda_{1}(D) \tau_{B}\right)$. Of course (2.10) is a standard fact in the theory of $q$-harmonic functions for the $\alpha$-stable Schrödinger operators. For us this will be a key formula for proving the Harnack inequality for $\varphi_{1}$.

By the well known formula for the distribution of the harmonic measure [26] we have

$$
E^{z} \varphi_{1}\left(X\left(\tau_{B}\right)\right)=\int_{B} G_{B}(z, y) \int_{D \backslash B}(2 \pi)^{-1}|y-w|^{-3} \varphi_{1}(w) d w d y
$$

To obtain our Harnack inequality for $\varphi_{1}$ we will first compare (2.10) and (2.11) and then we will use the formula for $E^{z} \varphi_{1}\left(X\left(\tau_{B}\right)\right)$. In order to compare (2.10) and (2.11) we need to compare $V_{B}(z, y)$ and $G_{B}(z, y)$. This will be done in a sequence of lemmas.

Lemma 2.4. Let $B \subsetneq D$ be any ball. Then for any $z, y \in B$ and $t_{0}>0$ we have

$$
V_{B}(z, y) \leq e^{\lambda_{1}(D) t_{0}} \int_{0}^{t_{0}} p_{B}(t, z, y) d t+\frac{1}{2 \pi(1-\beta)^{2} t_{0}}
$$

where $\beta=\lambda_{1}(D) / \lambda_{1}(B)$. 
Proof. We have

$$
V_{B}(z, y) \leq e^{\lambda_{1}(D) t_{0}} \int_{0}^{t_{0}} p_{B}(t, z, y) d t+\int_{t_{0}}^{\infty} e^{\lambda_{1}(D) t} p_{B}(t, z, y) d t .
$$

By (2.9) we obtain

$$
p_{B}(t, z, y)=\sum_{n=1}^{\infty} e^{-\lambda_{n}(B) t} \varphi_{n, B}(z) \varphi_{n, B}(y) \leq \frac{1}{2} \sum_{n=1}^{\infty} e^{-\lambda_{n}(B) t}\left(\varphi_{n, B}^{2}(z)+\varphi_{n, B}^{2}(y)\right) .
$$

It follows that the second integral in (2.12) is bounded above by

$$
\frac{1}{2} \int_{t_{0}}^{\infty} \sum_{n=1}^{\infty} e^{\left(\lambda_{1}(D)-\beta \lambda_{n}(B)\right) t} e^{-\lambda_{n}(B)(1-\beta) t}\left(\varphi_{n, B}^{2}(z)+\varphi_{n, B}^{2}(y)\right) d t
$$

where $\beta=\lambda_{1}(D) / \lambda_{1}(B)$. Since $\lambda_{1}(D)<\lambda_{1}(B)$ we have $\beta \in(0,1)$. Note also that $e^{\lambda_{1}(D)-\beta \lambda_{n}(B)} \leq e^{\lambda_{1}(D)-\beta \lambda_{1}(B)}=e^{0}=1$.

For any $w \in B(w=z$ or $w=y)$ we have

$$
\begin{aligned}
& \int_{t_{0}}^{\infty} \sum_{n=0}^{\infty} e^{-\lambda_{n}(B)(1-\beta) t} \varphi_{n, B}^{2}(w) d t=\int_{t_{0}}^{\infty} p_{B}((1-\beta) t, w, w) d t \\
& \leq \int_{t_{0}}^{\infty} p((1-\beta) t, 0,0) d t=\int_{t_{0}}^{\infty} \frac{1}{2 \pi(1-\beta)^{2} t^{2}} d t=\frac{1}{2 \pi(1-\beta)^{2} t_{0}} .
\end{aligned}
$$

We have used the formula for $p(t, 0,0)$ (see (1.2)). It follows that (2.13) is bounded from above by $1 /\left(2 \pi(1-\beta)^{2} t_{0}\right)$.

Lemma 2.5. Let $B=B(w, r), r>0, w \in \mathbb{R}^{2}$. For any $y \in B$ and $z \in B(w, r / 3)$ we have

$$
E^{y}\left(\tau_{B}\right) \leq\left(32 \sqrt{2} r^{2} / 3\right) G_{B}(z, y) .
$$

Proof. We may and do assume that $w=0$. It is well known ([15] formula $(2.10))$ that $E^{y}\left(\tau_{B}\right)=(2 / \pi)\left(r^{2}-|y|^{2}\right)^{1 / 2}$. It is also well known (see [13]) that

$$
G_{B(0,1)}(z, y)=\frac{1}{2 \pi^{2}|z-y|} \int_{0}^{w(z, y)} \frac{d r}{r^{1 / 2}(r+1)}, \quad z, y \in B(0,1),
$$

where $w(z, y)=\left(1-|z|^{2}\right)\left(1-|y|^{2}\right) /|z-y|^{2}$.

Note that for $t>0$ (by substituting $s=r^{1 / 2}$ )

$$
\int_{0}^{t} \frac{d r}{r^{1 / 2}(r+1)}=2 \arctan \left(t^{1 / 2}\right)
$$


Now, for $t \in(0,1]$ we have $2 \arctan \left(t^{1 / 2}\right) \geq 2 t^{1 / 2} \arctan (1)=t^{1 / 2} \pi / 2$, so for any $t>0$ we have $2 \arctan t^{1 / 2} \geq(\pi / 2)\left(t^{1 / 2} \wedge 1\right)$ where $a \wedge b$ denotes $\min (a, b)$. Hence $G_{B(0,1)}(z, y) \geq(4 \pi|z-y|)^{-1}\left(1 \wedge(w(z, y))^{1 / 2}\right)$. By scaling it follows that for any $z, y \in B=B(0, r)$,

$$
\begin{aligned}
G_{B}(z, y) & =r^{-1} G_{B(0,1)}\left(\frac{z}{r}, \frac{y}{r}\right) \\
& \geq \frac{1}{4 \pi r\left|\frac{z}{r}-\frac{y}{r}\right|}\left(1 \wedge \frac{\left(1-\left|\frac{z}{r}\right|^{2}\right)^{1 / 2}\left(1-\left|\frac{y}{r}\right|^{2}\right)^{1 / 2}}{\left|\frac{z}{r}-\frac{y}{r}\right|}\right) \\
& =\frac{1}{4 \pi r|z-y|}\left(r \wedge \frac{\left(r^{2}-|z|^{2}\right)^{1 / 2}\left(r^{2}-|y|^{2}\right)^{1 / 2}}{|z-y|}\right) .
\end{aligned}
$$

But for $z \in B(0, r / 3)$ and $y \in B(0, r)$ we have $|z-y| \leq 4 r / 3$ and $\left(r^{2}-\right.$ $\left.|z|^{2}\right)^{1 / 2} \geq(8 / 9)^{1 / 2} r$. Hence $\left(r^{2}-|z|^{2}\right)^{1 / 2}|z-y|^{-1} \geq 1 / \sqrt{2}$. It follows that for $z \in B(0, r / 3)$ and $y \in B(0, r),(2.14)$ is bounded below by

$$
\frac{3\left(r^{2}-|y|^{2}\right)^{1 / 2}}{16 \sqrt{2} \pi r^{2}}=\frac{3 E^{y}\left(\tau_{B}\right)}{32 \sqrt{2} r^{2}}
$$

and this completes the proof.

Lemma 2.6. Assume that $B=B(x, 3 / 8) \subsetneq D$. Then for any $z \in B(x, 1 / 8)$ and $y \in B$ we have $G_{B}(z, y) \leq V_{B}(z, y) \leq 16.6 G_{B}(z, y)$.

Proof. The inequality $G_{B}(z, y) \leq V_{B}(z, y)$ is trivial, it follows from the definition of $G_{B}(z, y)$ and $V_{B}(z, y)$.

We will prove the inequality $V_{B}(z, y) \leq 16.6 G_{B}(z, y)$. By Lemma 4.8 in [14] we have

$$
V_{B}(z, y)=G_{B}(z, y)+\lambda_{1}(D) \int_{B} V_{B}(z, u) G_{B}(u, y) d u .
$$

By Lemma 2.4, $\int_{B} V_{B}(z, u) G_{B}(u, y) d u$ is bounded above by

$$
e^{\lambda_{1}(D) t_{0}} \int_{B} \int_{0}^{t_{0}} p_{B}(t, z, u) d t G_{B}(u, y) d u+\frac{1}{2 \pi(1-\beta)^{2} t_{0}} \int_{B} G_{B}(u, y) d u
$$

where $\beta=\lambda_{1}(D) / \lambda_{1}(B)$. Let us denote the above sum by I + II. We have

$$
\begin{aligned}
& \int_{B} \int_{0}^{t_{0}} p_{B}(t, z, u) d t G_{B}(u, y) d u=\int_{0}^{t_{0}} \int_{0}^{\infty} \int_{B} p_{B}(t, z, u) p_{B}(s, u, y) d u d s d t \\
& =\int_{0}^{t_{0}} \int_{0}^{\infty} p_{B}(t+s, z, y) d s d t \leq t_{0} G_{B}(z, y) .
\end{aligned}
$$


It follows that $\mathrm{I} \leq t_{0} e^{\lambda_{1}(D) t_{0}} G_{B}(z, y)$.

By applying Lemma 2.5 for $r=3 / 8$ and $z \in B(x, 1 / 8)$ we get

$$
\mathrm{II}=\frac{E^{y}\left(\tau_{B}\right)}{2 \pi(1-\beta)^{2} t_{0}} \leq \frac{3 \sqrt{2} G_{B}(z, y)}{4 \pi(1-\beta)^{2} t_{0}} .
$$

Putting the estimates $(2.15),(2.16)$ together with those for I and II gives

$$
V_{B}(z, y) \leq G_{B}(z, y)\left(1+\lambda_{1}(D) t_{0} e^{\lambda_{1}(D) t_{0}}+\frac{3 \sqrt{2} \lambda_{1}(D)}{4 \pi(1-\beta)^{2} t_{0}}\right) .
$$

By our geometric assumptions on $D$, the inradius of $D$ is no smaller than $\sqrt{2} / 2$. Hence $\lambda_{1}(D) \leq \lambda_{1}(B(0, \sqrt{2} / 2))=\sqrt{2} \lambda_{1}(B(0,1))$. By scaling we also have $\lambda_{1}(B)=(8 / 3) \lambda_{1}(B(0,1))$ so $\beta=\lambda_{1}(D) / \lambda_{1}(B) \leq 3 \sqrt{2} / 8$. Hence, $(1-\beta)^{-2} \leq(1-3 \sqrt{2} / 8)^{-2}$. By $(2.15)$ in $[5], \lambda_{1}(B(0,1)) \leq 2 \pi / 3$ so $\lambda_{1}(D) \leq 2 \sqrt{2} \pi / 3$. By applying this to $(2.17)$ and putting $t_{0}=1 / 2$ we obtain $V_{B}(z, y) \leq 16.6 G_{B}(z, y)$, which completes the proof.

We now return to the proof of Theorem 2.3. Let $z_{1}, z_{2} \in B(x, 1 / 8) \subset$ $B(x, 3 / 8) \subset D$. By $(2.10),(2.11)$ and Lemma 2.6 we obtain

$$
\varphi_{1}\left(z_{2}\right) \geq E^{z_{2}}\left[\varphi_{1}\left(X\left(\tau_{B(x, 3 / 8)}\right)\right)\right]
$$

and

$$
\varphi_{1}\left(z_{1}\right) \leq 16.6 E^{z_{1}}\left[\varphi_{1}\left(X\left(\tau_{B(x, 3 / 8)}\right)\right)\right] .
$$

So to compare $\varphi_{1}\left(z_{2}\right)$ and $\varphi_{1}\left(z_{1}\right)$ we have to compare $E^{z_{1}}\left[\varphi_{1}\left(X\left(\tau_{B(x, 3 / 8)}\right)\right)\right]$ and $E^{z_{2}}\left[\varphi_{1}\left(X\left(\tau_{B(x, 3 / 8)}\right)\right)\right]$. Let $P_{r, x}(z, y)$ be the Poisson kernel for the ball $B(x, r) \subset \mathbb{R}^{2}$ for the Cauchy process. That is,

$$
P^{w}\left(X\left(\tau_{B(x, r)}\right) \in A\right)=\int_{A} P_{r, x}(z, y) d y
$$

where $w \in B(x, r), A \subset B^{c}(x, r)$. We have [13]

$$
P_{r, x}(z, y)=\frac{1}{\pi^{2}} \frac{\left(r^{2}-|z-x|^{2}\right)^{1 / 2}}{\left(|y-x|^{2}-r^{2}\right)^{1 / 2}|y-z|^{2}},
$$

$z \in B(x, r)$ and $y \in \operatorname{int}\left(B^{c}(x, r)\right)$. Put $r=3 / 8$. We have

$$
E^{z_{i}}\left[\varphi_{1}\left(X\left(\tau_{B(x, 3 / 8)}\right)\right)\right]=\int_{D \backslash \bar{B}} \varphi_{1}(y) P_{r, x}\left(z_{i}, y\right) d y
$$


for $i=1,2$. We have reduce to comparing $P_{r, x}\left(z_{1}, y\right)$ and $P_{r, x}\left(z_{2}, y\right)$. Recall that $z_{1}, z_{2} \in B(x, 1 / 8)$. For $y \in B^{c}(x, 3 / 8)$ we have

$$
\frac{\left|y-z_{2}\right|}{\left|y-z_{1}\right|} \leq 2 \quad \text { so } \quad \frac{\left|y-z_{2}\right|^{2}}{\left|y-z_{1}\right|^{2}} \leq 4
$$

and

$$
\frac{\left(r^{2}-\left|z_{1}-x\right|^{2}\right)^{1 / 2}}{\left(r^{2}-\left|z_{2}-x\right|^{2}\right)^{1 / 2}} \leq \frac{\left(r^{2}\right)^{1 / 2}}{\left(r^{2}-(r / 3)^{2}\right)^{1 / 2}}=\frac{3 \sqrt{2}}{4} .
$$

It follows that $P_{r, x}\left(z_{1}, y\right) / P_{r, x}\left(z_{2}, y\right) \leq 3 \sqrt{2}$. Using this, (2.20), (2.19) and (2.18) we obtain that for $z_{1}, z_{2} \in B(x, 1 / 8)$ we have $\varphi_{1}\left(z_{1}\right) \leq 16.6$. $3 \sqrt{2} \varphi_{1}\left(z_{2}\right)<70.5 \varphi_{1}\left(z_{2}\right)$.

\section{$3 \quad$ Weighted Poincaré inequalities}

The aim of this section is to prove Theorem 3.1 which gives a weighted Poincaré type inequality. This theorem will be a key step in proving our estimates for $\lambda_{2}-\lambda_{1}$ in $\S 4$. We will apply our Poincaré inequality to the function $g=\varphi_{1}^{2}$, where $\varphi_{1}$ is the first eigenfunction for the Cauchy semigroup. Such weighted Poincaré type inequalities are well known in the literature (see e.g., [32]) under the assumption that $g$ is $\log$-concave. The novelty of Theorem 3.1 is that we do not assume that $g$ is log-concave. Instead, we assume that $g$ is symmetric and unimodal with respect to both coordinate axis, that it is continuous, strictly positive and that it satisfies an appropriate Harnack inequality. These hypothesis replaces the log-concavity assumption. $\log$-concavity for the function $\varphi_{1}$ remains an interesting open question.

The proof of Theorem 3.1 is quite technical. Generally speaking, this is so because we can only efficiently control the function $g$ in the center of the domain $D$ where the Harnack inequality holds. One may ask whether Theorem 3.1 is true without the assumption that $g$ satisfies the Harnack inequality with a constant $C_{H}$. The answer is negative as we shall see later (Example 3.1).

A remark is perhaps in order here concerning the constants that appear in the formulation and in the proof of the Theorem 3.1. Our goal was to obtain a reasonable concrete constant in the conclusion of the Theorem 3.1 and at the same time emphasise our method rather than to strive for best constants which which these techniques do not have a hope of providing. In the case when the function $g$ is $\log$-concave, the inequality holds with $C_{P}=\pi^{2} / 8$, see for example [32] (note that the diameter $d_{D}$ of $D$ satisfies $\left.d_{D} \leq 2 \sqrt{2} L\right)$. 
Theorem 3.1. Let $D \subset \mathbb{R}^{2}$ satisfy the assumptions of Theorem 1.1. We assume that $g: D \rightarrow \mathbb{R}$ satisfies the following conditions.

(i) $g$ is continuous and strictly positive.

(ii) $g$ is symmetric with respect to both coordinate axes. That is, $g$ satisfies $g\left(x_{1},-x_{2}\right)=g\left(-x_{1}, x_{2}\right)=g\left(-x_{1},-x_{2}\right)=g\left(x_{1}, x_{2}\right)$.

(iii) $g$ is unimodal with respect to both coordinate axes as defined in part (iii) of Theorem 2.1.

(iv) If $B\left(x_{0}, 3 / 8\right) \subset D$, then on $B\left(x_{0}, 1 / 8\right) g$ satisfies the Harnack inequality with a constant $C_{H} \geq 1$. That is, for any $x, y \in B\left(x_{0}, 1 / 8\right)$ we have $g(x) \leq C_{H} g(y)$.

Suppose that $f \in C^{1}(D)$ is bounded and symmetric with respect to both coordinate axes and that

$$
\int_{D} f(x) g(x) d x=0
$$

Then we have

$$
\int_{D}|\nabla f(x)|^{2} g(x) d x \geq \frac{C_{P}}{L^{2}} \int_{D} f^{2}(x) g(x) d x
$$

where

$$
C_{P}=\min \left(\frac{16}{10^{5}}, \frac{2}{10^{2} C_{H}}\right)
$$

Throughout this section we will use the following notation. The function $p:(-1,1) \rightarrow(0, L]$ is defined so that for any $a_{2} \in(-1,1)$ we have $\left(p\left(a_{2}\right), a_{2}\right) \in \partial D$. Similarly, $r:(-L, L) \rightarrow(0,1]$ is defined so that for any $a_{1} \in(-L, L)$ we have $\left(a_{1}, r\left(a_{1}\right)\right) \in \partial D$. Set

$$
\tilde{D}=\left\{\left(x_{1}, x_{2}\right) \in D: x_{1} \geq 0, x_{2} \geq 0\right\} .
$$

Since $f$ and $g$ are symmetric with respect to both axis we can consider $\int_{\tilde{D}}|\nabla f| g$ instead of $\int_{D}|\nabla f| g$. Note that in particular we have $\int_{\tilde{D}} f g=0$. We also write $\tilde{D}=D_{1} \cup D_{2} \cup D_{3} \cup D_{4}$ where

$$
\begin{aligned}
& D_{1}=\left\{\left(x_{1}, x_{2}\right) \in \tilde{D}: x_{1} \leq L / 4, x_{2} \leq 1 / 4\right\}, \\
& D_{2}=\left\{\left(x_{1}, x_{2}\right) \in \tilde{D}: x_{1}>L / 4, x_{2} \leq 1 / 4\right\}, \\
& D_{3}=\left\{\left(x_{1}, x_{2}\right) \in \tilde{D}: x_{1} \leq L / 4, x_{2}>1 / 4\right\}, \\
& D_{4}=\left\{\left(x_{1}, x_{2}\right) \in \tilde{D}: x_{1}>L / 4, x_{2}>1 / 4\right\} .
\end{aligned}
$$


The proof of Theorem 3.1 will require several auxiliary lemmas. First, for any function $f$ we set

$$
f_{+}(x)=\left\{\begin{array}{rll}
f(x), & \text { when } & f(x) \geq 0 \\
0, & \text { when } & f(x)<0
\end{array}\right.
$$

and

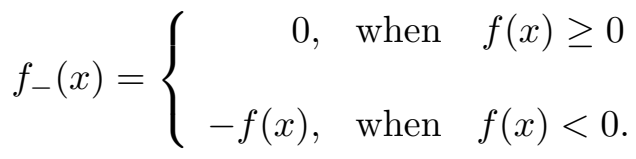

For brevity, we will write $f_{+}{ }^{\prime}(x)$ for $\left(f_{+}(x)\right)^{\prime}$.

Note that if $f \in C^{1}[a, b]$ then $f_{+}^{\prime}\left(x_{0}\right)$ is well defined except only for those $x_{0}$ for which $f\left(x_{0}\right)=0$ and such that $f(x)$ does not vanish in some neighborhood of $x_{0}$. Therefore there are at most countably many points on $(a, b)$ for which $f_{+}{ }^{\prime}$ does not exist. Note also that for any $a_{1}, a_{2} \in[a, b]$, $a<b$, we have

$$
\int_{a_{1}}^{a_{2}} f_{+}^{\prime}(x) d x=f_{+}\left(a_{2}\right)-f_{+}\left(a_{1}\right) .
$$

Similar remarks apply to $f_{-}$.

Lemma 3.2. Let $g:[a, b) \rightarrow \mathbb{R}$ be continuous, non-increasing and strictly positive. Let $f \in C^{1}[a, b)$ and $f(a)=0$. Then,

$$
\int_{a}^{b}\left(f_{+}^{\prime}(x)\right)^{2} g(x) d x \geq \frac{1}{(b-a)^{2}} \int_{a}^{b} f_{+}^{2}(x) g(x) d x .
$$

The same inequality holds if we replace $f_{+}$by $f_{-}$or $f$.

Proof. Let $M=\sup _{x \in[a, b)} f_{+}^{2}(x) g(x)$. We consider the following 2 cases:

Case 1: There exists $x_{0} \in[a, b)$ such that $f_{+}^{2}\left(x_{0}\right) g\left(x_{0}\right)=M$.

Case 2: There exists $\left\{x_{n}\right\} \subset[a, b), x_{n} \rightarrow b$, such that $f_{+}^{2}\left(x_{n}\right) g\left(x_{n}\right) \rightarrow M$.

First we deal with Case 1. We have

$$
\begin{aligned}
\int_{a}^{b}\left(f_{+}^{\prime}(x)\right)^{2} g(x) d x & \geq \int_{a}^{x_{0}}\left(f_{+}{ }^{\prime}(x)\right)^{2} g(x) d x \\
& \geq g\left(x_{0}\right) \int_{a}^{x_{0}}\left(f_{+}{ }^{\prime}(x)\right)^{2} d x .
\end{aligned}
$$


By Schwarz inequality this is larger than or equal to

$$
\begin{aligned}
\frac{g\left(x_{0}\right)}{x_{0}-a}\left(\int_{a}^{x_{0}}\left(f_{+}^{\prime}(x)\right) d x\right)^{2} & =\frac{g\left(x_{0}\right)}{x_{0}-a}\left(f_{+}\left(x_{0}\right)-f_{+}(a)\right)^{2} \\
& =\frac{f_{+}^{2}\left(x_{0}\right) g\left(x_{0}\right)}{x_{0}-a} \\
& \geq \frac{1}{(b-a)^{2}} \int_{a}^{b} f_{+}^{2}(x) g(x) d x .
\end{aligned}
$$

Next, consider Case 2. Arguing as in Case 1 we have

$$
\int_{a}^{b}\left(f_{+}^{\prime}(x)\right)^{2} g(x) d x \geq \frac{f_{+}^{2}\left(x_{n}\right) g\left(x_{n}\right)}{x_{n}-a} .
$$

By letting $n \rightarrow \infty$ we obtain

$$
\int_{a}^{b}\left(f_{+}^{\prime}(x)\right)^{2} g(x) d x \geq \frac{M}{b-a} \geq \frac{1}{(b-a)^{2}} \int_{a}^{b} f_{+}^{2}(x) g(x) d x .
$$

The proof for $f_{-}$or $f$ is similar.

Lemma 3.3. Let $f \in C^{1}[a, b)$ and $f(a)=0$. Then,

$$
\int_{a}^{b}\left(f_{+}{ }^{\prime}(x)\right)^{2} d x \geq \frac{1}{(b-a)^{2}} \int_{a}^{b} f_{+}^{2}(x) d x .
$$

The same inequality holds if we replace $f_{+}$by $f_{-}$or by $f$. Also, the same conclusion is true when we assume that $f \in C^{1}(a, b]$ and $f(b)=0$.

Proof. This follows from Lemma 3.2 with $g \equiv 1$.

Lemma 3.4. Let $a<b, g:[a, b) \rightarrow \mathbb{R}$ continuous, non-increasing, strictly positive and let $f \in C^{1}[a, b)$. Let $\eta \in(0,1)$ and $A>0$. Assume that $f(a) \leq A \eta$. Put $F=\{x \in[a, b): f(x) \geq A\}$. Then,

$$
\int_{a}^{b}\left(f_{+}^{\prime}(x)\right)^{2} g(x) d x \geq \frac{(1-\eta)^{2}}{(b-a)^{2}} \int_{F} f_{+}^{2}(x) g(x) d x .
$$

The same inequality holds if we replace $f_{+}$by $f$.

Proof. We can and do assume that $F$ is not empty. That is, there exists $x \in(a, b)$ such that $f(x) \geq A$. Let $h(x)=f(x)-A \eta$. We have $h(a) \leq 0$. Put

$$
a_{1}=\inf \{x \in[a, b): f(x)=A \eta\}=\inf \{x \in[a, b): h(x)=0\} .
$$


For $x \in[a, b)$ such that $f(x) \geq A \eta$ we have ${f_{+}}^{\prime}(x)=h_{+}{ }^{\prime}(x)$ so

$$
\int_{a}^{b}\left(f_{+}{ }^{\prime}(x)\right)^{2} g(x) d x \geq \int_{a_{1}}^{b}\left(h_{+}{ }^{\prime}(x)\right)^{2} g(x) d x .
$$

By Lemma 3.2 this is bounded below by

$$
\frac{1}{\left(b-a_{1}\right)^{2}} \int_{a_{1}}^{b} h_{+}^{2}(x) g(x) d x .
$$

Note that $\left[a, a_{1}\right] \cap F$ is empty and that for $x \in F$ we have $h_{+}(x)=f_{+}(x)-$ $A \eta \geq(1-\eta) f_{+}(x)$. It follows that $(3.3)$ is bounded below by

$$
\frac{(1-\eta)^{2}}{(b-a)^{2}} \int_{F} f_{+}^{2}(x) g(x) d x
$$

and this completes the proof.

Lemma 3.5. Let $a<b, f \in C^{1}(a, b], \eta \in(0,1)$ and $A>0$. Assume that $f(b) \leq A \eta$. Put $F=\{x \in(a, b]: f(x) \geq A\}$. Then

$$
\int_{a}^{b}\left(f_{+}{ }^{\prime}(x)\right)^{2} d x \geq \frac{(1-\eta)^{2}}{(b-a)^{2}} \int_{F} f_{+}^{2}(x) d x .
$$

The same inequality holds if we replace $f_{+}$by $f$.

Proof. This follows from Lemma 3.4 with $g \equiv 1$.

Lemma 3.6. Let $0<a_{1}<a_{2}$. Suppose $g:\left[0, a_{2}\right) \rightarrow \mathbb{R}$ is continuous, non-increasing, strictly positive and let $f \in C^{1}\left[0, a_{2}\right)$. Fix $\delta_{1} \in(0,1)$. Then at least one of the following inequalities holds

$$
\begin{gathered}
\int_{0}^{a_{1}} f_{+}^{2}(x) g(x) d x \geq \delta_{1} \frac{a_{1}}{a_{2}} \int_{0}^{a_{2}} f_{+}^{2}(x) g(x) d x \\
\int_{0}^{a_{2}}\left(f_{+}^{\prime}(x)\right)^{2} g(x) d x \geq \frac{\left(1-\sqrt{\delta_{1}}\right)^{2}}{a_{2}^{2}} \int_{0}^{a_{2}} f_{+}^{2}(x) g(x) d x .
\end{gathered}
$$

The same conclusion holds if in (3.4) and (3.5) we replace $f_{+}$with $f_{-}$or $f$. Proof. Assume that

$$
\int_{0}^{a_{1}} f_{+}^{2}(x) g(x) d x<\delta_{1} \frac{a_{1}}{a_{2}} \int_{0}^{a_{2}} f_{+}^{2}(x) g(x) d x .
$$


Let

$$
M=\sup _{x \in\left[a_{1}, a_{2}\right)} f_{+}^{2}(x) g(x)
$$

and $x_{1} \in\left[0, a_{1}\right]$ be such that

$$
f_{+}^{2}\left(x_{1}\right) g\left(x_{1}\right)=\min _{x \in\left[0, a_{1}\right]} f_{+}^{2}(x) g(x) .
$$

Let $C=\int_{0}^{a_{2}} f_{+}^{2} g$. We will show that

$$
f_{+}^{2}\left(x_{1}\right) g\left(x_{1}\right) \leq \frac{C \delta_{1}}{a_{2}}
$$

and

$$
M \geq \frac{C}{a_{2}}
$$

Observe that (3.7) follows easily from (3.6). To show (3.8) note that by (3.6) we have

$$
\int_{0}^{a_{1}} f_{+}^{2} g<\delta_{1} C a_{1} / a_{2} .
$$

If (3.8) does not hold we would have

$$
\int_{a_{1}}^{a_{2}} f_{+}^{2} g<C\left(a_{2}-a_{1}\right) / a_{2}
$$

so

$$
\int_{0}^{a_{2}} f_{+}^{2} g<C
$$

which gives contradiction and hence (3.8) holds.

Let $\varepsilon \in(0,1)$ and let $x_{0} \in\left[a_{1}, a_{2}\right)$ be such that $f_{+}^{2}\left(x_{0}\right) g\left(x_{0}\right) \geq M(1-\varepsilon)$. (3.8) gives that $f_{+}^{2}\left(x_{0}\right) g\left(x_{0}\right) \geq C(1-\varepsilon) / a_{2}$. Note also that

$$
f_{+}^{2}\left(x_{1}\right) \leq \frac{\delta_{1} C}{a_{2} g\left(x_{1}\right)} \leq \frac{\delta_{1} C}{a_{2} g\left(x_{0}\right)} \leq \frac{f_{+}^{2}\left(x_{0}\right) \delta_{1}}{1-\varepsilon},
$$

so $f_{+}\left(x_{1}\right) \leq f_{+}\left(x_{0}\right) \sqrt{\delta_{1}} / \sqrt{1-\varepsilon}$. 
Now let $\varepsilon \in\left(0,1-\delta_{1}\right)$, so $\delta_{1} /(1-\varepsilon)<1$. We have

$$
\begin{aligned}
\int_{0}^{a_{2}}\left(f_{+}^{\prime}(x)\right)^{2} g(x) d x & \geq g\left(x_{0}\right) \int_{x_{1}}^{x_{0}}\left(f_{+}^{\prime}(x)\right)^{2} d x \\
& \geq \frac{g\left(x_{0}\right)}{x_{0}-x_{1}}\left(f_{+}\left(x_{0}\right)-f_{+}\left(x_{1}\right)\right)^{2} \\
& \geq \frac{f^{2}\left(x_{0}\right) g\left(x_{0}\right)}{x_{0}-x_{1}}\left(1-\frac{\sqrt{\delta_{1}}}{\sqrt{1-\varepsilon}}\right)^{2} \\
& \geq \frac{C(1-\varepsilon)}{a_{2}^{2}}\left(1-\frac{\sqrt{\delta_{1}}}{\sqrt{1-\varepsilon}}\right)^{2}
\end{aligned}
$$

By letting $\varepsilon \rightarrow 0$, we obtain (3.5).

Lemma 3.7. Let $a<b$. Suppose $g:[a, b) \rightarrow(0, \infty)$ is continuous and non-increasing and let $f \in C^{1}[a, b)$. Let $\eta \in(0,1)$ and $A>0$. Assume that $f(a) \geq A \eta^{2}$. Then at least one of the following inequalities holds

$$
\begin{gathered}
\int_{a}^{b} f^{2}(x) g(x) d x \geq \frac{A^{2} \eta^{6}}{2} \int_{a}^{b} g(x) d x, \\
\int_{a}^{b}\left(f^{\prime}(x)\right)^{2} g(x) d x \geq \frac{A^{2} \eta^{4}(1-\eta)^{2}}{2(b-a)^{2}} \int_{a}^{b} g(x) d x .
\end{gathered}
$$

Proof. Put $M=\int_{a}^{b} g(x) d x$. If there exists $x \in[a, b)$ such that $f(x)=A \eta^{3}$ let $x_{0}=\min \left\{x \in[a, b): f(x)=A \eta^{3}\right\}$, in the other case let $x_{0}=b$. If

$$
\int_{a}^{x_{0}} g(x) d x \geq M / 2
$$

then we have

$$
\int_{a}^{x_{0}} f^{2}(x) g(x) d x \geq \frac{A^{2} \eta^{6} M}{2},
$$

which gives (3.9). If

$$
\int_{a}^{x_{0}} g(x) d x<M / 2
$$

then

$$
(b-a) g\left(x_{0}\right) \geq \int_{x_{0}}^{b} g(x) d x>\frac{M}{2},
$$


so $g\left(x_{0}\right) \geq M /(2(b-a))$. It follows that

$$
\begin{aligned}
\int_{a}^{x_{0}}\left(f^{\prime}(x)\right)^{2} g(x) d x \geq g\left(x_{0}\right) \int_{a}^{x_{0}}\left(f^{\prime}(x)\right)^{2} d x & \geq \frac{M\left(f(a)-f\left(x_{0}\right)\right)^{2}}{2(b-a)\left(x_{0}-a\right)} \\
& \geq \frac{M A^{2} \eta^{4}(1-\eta)^{2}}{2(b-a)^{2}}
\end{aligned}
$$

which gives (3.10).

Lemma 3.8. Let $D$ and $g$ be as in Theorem 3.1. Then the following Harnack inequalities hold

$$
\begin{aligned}
& \forall x_{1} \in[0, L / 4], \quad \forall x_{2} \in[0,1 / 4], \quad g\left(x_{1}, x_{2}\right) \geq g\left(x_{1}, 0\right) / C_{H}, \\
& \forall x_{2} \in[0,1 / 4], \quad \forall x_{1} \in[0,1 / 4], \quad g\left(x_{1}, x_{2}\right) \geq g\left(0, x_{2}\right) / C_{H} .
\end{aligned}
$$

Proof. Consider the rhombus $R=\left\{\left(x_{1}, x_{2}\right): x_{1} \in(-L, L), x_{2} \in(1-\right.$ $\left.\left.\left|x_{1}\right| / L,\left|x_{1}\right| / L-1\right)\right\}$. By our assumptions on $D, R \subset D$. It also follows that for all $x_{1} \in[0, L / 4] B\left(\left(x_{1}, 1 / 8\right), 3 / 8\right) \subset R \subset D$. Therefore for all

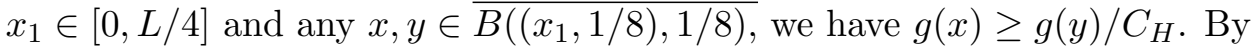
putting $x=\left(x_{1}, x_{2}\right), y=\left(x_{1}, 0\right)$ we get $(3.11)$.

In a similar way, it follows that for all $x_{2} \in[0,1 / 4], B\left(\left(1 / 8, x_{2}\right), 3 / 8\right) \subset$ $R \subset D$ and this implies, as above, (3.12).

Lemma 3.9. Let $D, f$ and $g$ be as in Theorem 3.1. Fix $\delta_{1}, \delta_{2} \in(0,1)$. Then at least one of the following inequalities holds

$$
\int_{D_{1} \cup D_{2}} f_{+}^{2} g \geq \frac{\delta_{1} \delta_{2}}{4} \int_{\tilde{D}} f_{+}^{2} g
$$

or

$$
\int_{\tilde{D}}\left(\frac{\partial f_{+}}{\partial x_{2}}\right)^{2} g \geq\left(1-\sqrt{\delta_{1}}\right)^{2}\left(1-\delta_{2}\right) \int_{\tilde{D}} f_{+}^{2} g
$$

The same conclusion holds if in (3.13) and (3.14) we replace $f_{+}$with $f_{-}$or $f$.

Proof. Recall that $r:(-L, L) \rightarrow(0,1]$ is such that $\left(x_{1}, r\left(x_{1}\right)\right) \in \partial D, x_{1} \in$ $(-L, L)$ and let $a \wedge b$ denote the minimum of $a$ and $b$. By Lemma 3.6 for any $x_{1} \in[0, L)$ at least one of the following inequalities holds

$$
\begin{aligned}
& \int_{0}^{(1 / 4) \wedge r\left(x_{1}\right)}\left(f_{+}\left(x_{1}, x_{2}\right)\right)^{2} g\left(x_{1}, x_{2}\right) d x_{2} \geq \\
& \delta_{1} \frac{(1 / 4) \wedge r\left(x_{1}\right)}{r\left(x_{1}\right)} \int_{0}^{r\left(x_{1}\right)} f_{+}^{2}\left(x_{1}, x_{2}\right) g\left(x_{1}, x_{2}\right) d x_{2},
\end{aligned}
$$


or

$$
\begin{aligned}
& \int_{0}^{r\left(x_{1}\right)}\left(\frac{\partial f_{+}}{\partial x_{2}}\right)^{2}\left(x_{1}, x_{2}\right) g\left(x_{1}, x_{2}\right) d x_{2} \geq \\
& \frac{\left(1-\sqrt{\delta_{1}}\right)^{2}}{r^{2}\left(x_{1}\right)} \int_{0}^{r\left(x_{1}\right)} f_{+}^{2}\left(x_{1}, x_{2}\right) g\left(x_{1}, x_{2}\right) d x_{2} .
\end{aligned}
$$

Let

$$
F=\left\{x_{1} \in[0, L):(3.15) \text { holds }\right\} \quad \text { and } \quad G=\left\{x_{1} \in[0, L):(3.16) \text { holds }\right\} .
$$

If

$$
\int_{F} \int_{0}^{r\left(x_{1}\right)} f_{+}^{2}\left(x_{1}, x_{2}\right) g\left(x_{1}, x_{2}\right) d x_{2} d x_{1} \geq \delta_{2} \int_{\tilde{D}} f_{+}^{2}\left(x_{1}, x_{2}\right) g\left(x_{1}, x_{2}\right) d x_{2} d x_{1}
$$

then (3.13) holds. In the other case, (3.14) holds and this completes the proof.

Lemma 3.10. Let $D, f$ and $g$ be as in Theorem 3.1. Fix $\delta_{1}, \delta_{2} \in(0,1)$. Then at least one of the following inequalities holds

$$
\int_{D_{1} \cup D_{3}} f_{+}^{2} g \geq \frac{\delta_{1} \delta_{2}}{4} \int_{\tilde{D}} f_{+}^{2} g
$$

or

$$
\int_{\tilde{D}}\left(\frac{\partial f_{+}}{\partial x_{1}}\right)^{2} g \geq \frac{\left(1-\sqrt{\delta_{1}}\right)^{2}\left(1-\delta_{2}\right)}{L^{2}} \int_{\tilde{D}} f_{+}^{2} g
$$

The same conclusion holds if in (3.17) and (3.18) we replace $f_{+}$with $f_{-}$or $f$.

The proof of this lemma is the same as the proof of Lemma 3.9 and it is omitted.

Lemma 3.11. Let $D, f$ and $g$ be as in Theorem 3.1. Assume additionally that $\int_{D} f^{2} g=1$ and $\int_{D} g=1$. Fix $\eta \in(0,1)$ and let $A=4 \sqrt{2} / \eta^{3}$. Assume there exists $a \in[0,1 / 8]$ such that for all $x_{2} \in[0,1 / 4], f\left(a, x_{2}\right) \geq A \eta^{2}$. Then,

$$
\int_{\tilde{D}}|\nabla f|^{2} g \geq \min \left(\frac{(1-\eta)^{2}}{4 \eta^{2} L^{2}}, \frac{32(1-\eta)^{2}}{\eta^{2} C_{H}}\right) .
$$


Proof. We assume that $\int_{D} g=1$. In particular, by symmetry $\int_{\tilde{D}} g=1 / 4$. By monotonicity properties of $g$ we have $\int_{D_{1} \cup D_{2}} g \geq(1 / 4) \int_{\tilde{D}} g=1 / 16$. Let $R=[0, a] \times[0,1 / 4]$. We will consider 2 cases:

\section{Case 1.}

$$
\int_{\left(D_{1} \cup D_{2}\right) \backslash R} g \geq \frac{1}{32}
$$

\section{Case 2.}

$$
\int_{R} g \geq \frac{1}{32}
$$

At first let us consider Case 1. By Lemma 3.7 for any $x_{2} \in[0,1 / 4]$ at least one of the following inequalities holds

$$
\int_{a}^{p\left(x_{2}\right)} f^{2}\left(x_{1}, x_{2}\right) g\left(x_{1}, x_{2}\right) d x_{1} \geq \frac{A^{2} \eta^{6}}{2} \int_{a}^{p\left(x_{2}\right)} g\left(x_{1}, x_{2}\right) d x_{1},
$$

$$
\int_{a}^{p\left(x_{2}\right)}\left(\frac{\partial f}{\partial x_{1}}\left(x_{1}, x_{2}\right)\right)^{2} g\left(x_{1}, x_{2}\right) d x_{1} \geq \frac{A^{2} \eta^{4}(1-\eta)^{2}}{2 L^{2}} \int_{a}^{p\left(x_{2}\right)} g\left(x_{1}, x_{2}\right) d x_{1} .
$$

Recall that $p:(-1,1) \rightarrow(0, L]$ is defined so that $\left(p\left(a_{2}\right), a_{2}\right) \in \partial D$ for $a_{2} \in(-1,1)$. Let

$$
F=\left\{x_{2} \in[0,1 / 4]:(3.22) \text { holds }\right\}, \quad G=\left\{x_{2} \in[0,1 / 4]:(3.23) \text { holds }\right\} .
$$

$F \cup G=[0,1 / 4]$ so by $(3.20)$ we have that at least one of the following inequalities holds

$$
\begin{aligned}
& \int_{F} \int_{a}^{p\left(x_{2}\right)} g\left(x_{1}, x_{2}\right) d x_{1} d x_{2}>\frac{1}{64}, \\
& \int_{G} \int_{a}^{p\left(x_{2}\right)} g\left(x_{1}, x_{2}\right) d x_{1} d x_{2} \geq \frac{1}{64} .
\end{aligned}
$$

Recall that we assume $\int_{D} f^{2} g=1$, so by symmetry $\int_{\tilde{D}} f^{2} g=1 / 4$. If (3.24) holds we would have (see definition of $F$ )

$$
\frac{1}{4}=\int_{\tilde{D}} f^{2} g \geq \int_{F} \int_{a}^{p\left(x_{2}\right)} f^{2}\left(x_{1}, x_{2}\right) g\left(x_{1}, x_{2}\right) d x_{1} d x_{2}>\frac{A^{2} \eta^{6}}{128}=\frac{1}{4},
$$


because $A=4 \sqrt{2} / \eta^{3}$. This gives a contradiction, so (3.24) cannot hold.

Hence (3.25) must hold. By the definition of $G$ it follows that

$$
\begin{aligned}
\int_{\tilde{D}}|\nabla f|^{2} g & \geq \int_{G} \int_{a}^{p\left(x_{2}\right)}\left(\frac{\partial f}{\partial x_{1}}\left(x_{1}, x_{2}\right)\right)^{2} g\left(x_{1}, x_{2}\right) d x_{1} d x_{2} \\
& \geq \frac{A^{2} \eta^{4}(1-\eta)^{2}}{128 L^{2}}=\frac{(1-\eta)^{2}}{4 \eta^{2} L^{2}} .
\end{aligned}
$$

This finishes Case 1.

Now let us consider Case 2. Let

$$
\begin{aligned}
& F=\left\{x_{2} \in[0,1 / 4]: \forall x_{1} \in[0, a] f\left(x_{1}, x_{2}\right)>A \eta^{3}\right\}, \\
& G=\left\{x_{2} \in[0,1 / 4]: \exists x_{1} \in[0, a] f\left(x_{1}, x_{2}\right) \leq A \eta^{3}\right\} .
\end{aligned}
$$

$F \cup G=[0,1 / 4]$ so by $(3.21)$ at least one of the following inequalities holds

$$
\begin{aligned}
& \int_{F} \int_{0}^{a} g\left(x_{1}, x_{2}\right) d x_{1} d x_{2}>\frac{1}{64}, \\
& \int_{G} \int_{0}^{a} g\left(x_{1}, x_{2}\right) d x_{1} d x_{2} \geq \frac{1}{64} .
\end{aligned}
$$

If (3.26) holds we would have

$$
\frac{1}{4}=\int_{\tilde{D}} f^{2} g \geq \int_{F} \int_{0}^{a} f^{2}\left(x_{1}, x_{2}\right) g\left(x_{1}, x_{2}\right) d x_{1} d x_{2}>\frac{A^{2} \eta^{6}}{64}=\frac{1}{2} .
$$

This gives a contradiction, so (3.27) must hold. For any $x_{2} \in G$, let $h\left(x_{2}\right)=$ $\min \left\{x_{1} \in[0, a]: f\left(x_{1}, x_{2}\right) \leq A \eta^{3}\right\}$. By the Harnack inequality (3.12) we obtain

$$
\begin{aligned}
\int_{\tilde{D}}|\nabla f|^{2} g & \geq \int_{G} \int_{0}^{a}\left(\frac{\partial f}{\partial x_{1}}\left(x_{1}, x_{2}\right)\right)^{2} g\left(x_{1}, x_{2}\right) d x_{1} d x_{2} \\
& \geq C_{H}^{-1} \int_{G} g\left(0, x_{2}\right) \int_{h\left(x_{2}\right)}^{a}\left(\frac{\partial f}{\partial x_{1}}\left(x_{1}, x_{2}\right)\right)^{2} d x_{1} d x_{2} \\
& \geq C_{H}^{-1} \int_{G} g\left(0, x_{2}\right) \frac{\left(f(a)-f\left(h\left(x_{2}\right)\right)\right)^{2}}{\left|a-h\left(x_{2}\right)\right|} d x_{2} .
\end{aligned}
$$


Note that $a \leq 1 / 8$ and $\left|a-h\left(x_{2}\right)\right| \leq 1 / 8$. It follows that the right hand side of (3.28) is bounded below by

$$
\begin{aligned}
& C_{H}^{-1} 8 A^{2} \eta^{4}(1-\eta)^{2} \int_{G} g\left(0, x_{2}\right) d x_{2} \\
& \geq C_{H}^{-1} 8 A^{2} \eta^{4}(1-\eta)^{2} a^{-1} \int_{G} \int_{0}^{a} g\left(x_{1}, x_{2}\right) d x_{1} d x_{2} \\
& \geq \frac{A^{2} \eta^{4}(1-\eta)^{2}}{C_{H}}=\frac{32(1-\eta)^{2}}{\eta^{2} C_{H}} .
\end{aligned}
$$

Lemma 3.12. Let $D, f$ and $g$ be as in Theorem 3.1. Assume in addition that $\int_{D} f^{2} g=1$ and $\int_{D} g=1$. Fix $\eta \in(0,1)$ and let $A=4 \sqrt{2} / \eta^{3}$. Assume there exists $b_{2} \in[0,1 / 4]$ such that for any $x_{1} \in[0,1 / 8], f\left(x_{1}, b_{2}\right) \geq A \eta$. Then,

$$
\int_{\tilde{D}}|\nabla f|^{2} g \geq \min \left(\frac{(1-\eta)^{2}}{4 \eta^{2} L^{2}}, \frac{4(1-\eta)^{2}}{\eta^{2} C_{H} L}\right) .
$$

Proof. If there is an $a \in[0,1 / 8]$ such that for all $x_{2} \in[0,1 / 4], f\left(a, x_{2}\right) \geq$ $A \eta^{2}$, then (3.29) follows from Lemma 3.11. In the other case, for all $x_{1} \in$ $[0,1 / 8]$ there is $x_{2} \in[0,1 / 4]$ such that $f\left(x_{1}, x_{2}\right)<A \eta^{2}$. For any $x_{1} \in[0,1 / 8]$ let

$$
h\left(x_{1}\right)=\min \left\{x_{2} \in[0,1 / 4]: f\left(x_{1}, x_{2}\right) \leq A \eta^{2}\right\} .
$$

Let $I\left(x_{1}\right)=\left[b_{2}, h\left(x_{1}\right)\right]$, if $h\left(x_{1}\right)>b_{2}$ and $I\left(x_{1}\right)=\left[h\left(x_{1}\right), b_{2}\right]$, if $h\left(x_{1}\right) \leq b_{2}$.

Recall that $\int_{\tilde{D}} g=1 / 4$. By the monotonicity properties of $g$ (in the $x_{2}$-direction) we have

$$
\int_{0}^{L} g\left(x_{1}, 0\right) d x_{1} \geq 1 / 4
$$

On the other hand, by the monotonicity properties of $g$ (in the $x_{1}$-direction) we get

$$
\int_{0}^{1 / 8} g\left(x_{1}, 0\right) d x_{1} \geq \frac{1}{8 L} \int_{0}^{L} g\left(x_{1}, 0\right) d x_{1} \geq \frac{1}{32 L} .
$$

By the Harnack inequality (3.11) we have that for all $x_{1} \in[0,1 / 8]$ and 
all $x_{2} \in[0,1 / 4], g\left(x_{1}, x_{2}\right) \geq C_{H}^{-1} g\left(x_{1}, 0\right)$. It follows that

$$
\begin{aligned}
& \int_{0}^{1 / 8} \int_{0}^{1 / 4}\left(\frac{\partial f}{\partial x_{2}}\right)^{2}\left(x_{1}, x_{2}\right) g\left(x_{1}, x_{2}\right) d x_{2} d x_{1} \\
& \geq \frac{1}{C_{H}} \int_{0}^{1 / 8} \int_{I\left(x_{1}\right)}\left(\frac{\partial f}{\partial x_{2}}\right)^{2}\left(x_{1}, x_{2}\right) d x_{2} g\left(x_{1}, 0\right) d x_{1} \\
& \geq \frac{1}{C_{H}} \int_{0}^{1 / 8} \frac{1}{\left|h\left(x_{1}\right)-b_{2}\right|}\left(f\left(x_{1}, h\left(x_{1}\right)\right)-f\left(x_{1}, b_{2}\right)\right)^{2} g\left(x_{1}, 0\right) d x_{1} \\
& \geq \frac{4\left(A \eta-A \eta^{2}\right)^{2}}{C_{H}} \int_{0}^{1 / 8} g\left(x_{1}, 0\right) d x_{1} \\
& \geq \frac{A^{2} \eta^{2}(1-\eta)^{2}}{8 C_{H} L}=\frac{4(1-\eta)^{2}}{C_{H} \eta^{4} L} .
\end{aligned}
$$

The last expression is greater than the right-hand side of (3.29). This proves the lemma.

Proof of Theorem 3.1. We assume, for the rest of the the proof, that $\int_{D} f^{2} g=$ 1 and $\int_{D} g=1$. In particular, by symmetry, $\int_{\tilde{D}} f^{2} g=1 / 4$ and $\int_{\tilde{D}} g=1 / 4$. Fix the constant $m=1 / 320$. Such a choice will become clear later. We will consider the following $\mathbf{5}$ cases:

Case 1. Suppose that

$$
\int_{D_{1} \cup D_{2}} f^{2} g<1 / 20 .
$$

Then,

$$
\int_{\tilde{D}}|\nabla f|^{2} g \geq \frac{7}{10^{5}}
$$

Case 2. Suppose that

$$
\int_{D_{1} \cup D_{2}} f^{2} g \geq 1 / 20
$$

and that

$$
\int_{\tilde{D}} f_{+}^{2} g<m \text { or that } \int_{\tilde{D}} f_{-}^{2} g<m
$$

Then,

$$
\int_{\tilde{D}}|\nabla f|^{2} g \geq \min \left(\frac{1}{10^{3}}, \frac{64}{10^{3} C_{H}}\right) \frac{1}{L^{2}}
$$


Case 3. Suppose that

$$
\int_{\tilde{D}} f_{+}^{2} g \geq m \text { and } \quad \int_{\tilde{D}} f_{-}^{2} g \geq m
$$

and that

$$
\min \left(\int_{D_{1} \cup D_{2}} f_{+}^{2} g, \int_{D_{1} \cup D_{2}} f_{-}^{2} g, \int_{D_{1} \cup D_{3}} f_{+}^{2} g, \int_{D_{1} \cup D_{3}} f_{-}^{2} g\right)<\frac{m}{10} .
$$

Then,

$$
\int_{\tilde{D}}|\nabla f|^{2} g \geq \frac{4}{10^{5} L^{2}}
$$

Case 4. Suppose that

$$
\min \left(\int_{D_{1} \cup D_{2}} f_{+}^{2} g, \int_{D_{1} \cup D_{2}} f_{-}^{2} g, \int_{D_{1} \cup D_{3}} f_{+}^{2} g, \int_{D_{1} \cup D_{3}} f_{-}^{2} g\right) \geq \frac{m}{10} .
$$

and that there exists an $x_{1} \in[0, L / 4]$ such that for all $x_{2} \in[0,1 / 4]$, $\left|f\left(x_{1}, x_{2}\right)\right|>0$. Then,

$$
\int_{\tilde{D}}|\nabla f|^{2} g \geq \min \left(\frac{1}{32 \cdot 10^{2}}, \frac{1}{2 \cdot 10^{2} C_{H}}\right) \frac{1}{L^{2}}
$$

Case 5. Suppose that

$$
\min \left(\int_{D_{1} \cup D_{2}} f_{+}^{2} g, \int_{D_{1} \cup D_{2}} f_{-}^{2} g, \int_{D_{1} \cup D_{3}} f_{+}^{2} g, \int_{D_{1} \cup D_{3}} f_{-}^{2} g\right) \geq \frac{m}{10} .
$$

and that for all $x_{1} \in[0, L / 4]$ there exists $x_{2} \in[0,1 / 4]$, such that $f\left(x_{1}, x_{2}\right)=$ 0 . Then,

$$
\int_{\tilde{D}}|\nabla f|^{2} g \geq \min \left(\frac{1}{32 \cdot 10^{2}}, \frac{1}{2 \cdot 10^{2} C_{H}}\right) .
$$

Before proving the inequalities in these five case, let us see how they imply the assertion of Theorem 3.1. By comparing constants in (3.31)(3.35) we get

$$
\int_{\tilde{D}}|\nabla f|^{2} g \geq \frac{1}{L^{2}} \min \left(\frac{4}{10^{5}}, \frac{1}{2 \cdot 10^{2} \cdot C_{H}}\right) .
$$

Now, the equalities

$$
\int_{\tilde{D}}|\nabla f|^{2} g=4 \int_{D}|\nabla f|^{2} g
$$


and

$$
\int_{D} f^{2} g=1
$$

give the assertion of Theorem 3.1.

We now return to the inequalities in Cases 1-5.

Case 1. We will use Lemma 3.9 with $f_{+}$replaced by $f$. Recall that $\int_{\tilde{D}} f^{2} g=$ $1 / 4$. Take $\delta_{1}=\sqrt{4 / 5}, \delta_{2}=\sqrt{4 / 5}$. (3.30) gives that (3.13) does not hold. We have $\left(1-\sqrt{\delta_{1}}\right)^{2}\left(1-\delta_{2}\right)(1 / 4) \geq 7 \cdot 10^{-5}$. (3.31) follows by (3.14) (with $f_{+}$replaced by $\left.f\right)$.

Case 2. For this we choose the following constants: $\eta=1 / \sqrt{2}, A=$ $4 \sqrt{2} / \eta^{3}=16$ and apply Lemma 3.12. Note also that $m=1 / 320$ is chosen so that $m=1 /(20 A)$. Since

$$
\int_{D_{1} \cup D_{2}} f^{2} g \geq 1 / 20
$$

we have

$$
\int_{D_{1} \cup D_{2}} f_{+}^{2} g \geq 1 / 40
$$

or

$$
\int_{D_{1} \cup D_{2}} f_{-}^{2} g \geq 1 / 40
$$

We may assume that

$$
\int_{D_{1} \cup D_{2}} f_{+}^{2} g \geq 1 / 40
$$

This and the assumption in Case 2 give

$$
\int_{\tilde{D}} f_{-}^{2} g<m
$$

Let

$$
F=\left\{x \in D_{1} \cup D_{2}: f_{+}(x) \leq A\right\}
$$

and

$$
G=\left\{x \in D_{1} \cup D_{2}: f_{+}(x)>A\right\} .
$$

We will show that

$$
\int_{F} f_{+}^{2} g<\frac{1}{2} \int_{D_{1} \cup D_{2}} f_{+}^{2} g .
$$


Since

$$
\int_{\tilde{D}} f g=0
$$

we have that

$$
\int_{\tilde{D}} f_{+} g=\int_{\tilde{D}} f_{-} g
$$

This gives

$$
\begin{aligned}
\int_{F} f_{+}^{2} g & \leq A \int_{F} f_{+} g \leq A \int_{\tilde{D}} f_{+} g \\
& =A \int_{\tilde{D}} f_{-} g \\
& \leq A \int_{\tilde{D}} f_{-}^{2} g \int_{\tilde{D}} g \\
& <\frac{A m}{4} .
\end{aligned}
$$

We have

$$
\int_{D_{1} \cup D_{2}} f_{+}^{2} g \geq 1 / 40
$$

and since $m=1 /(20 A)$ we have

$$
\int_{F} f_{+}^{2} g<\frac{A m}{4}=\frac{1}{80} \leq \frac{1}{2} \int_{D_{1} \cup D_{2}} f_{+}^{2} g,
$$

which gives (3.36). (We note that it is here where the concrete value of $m=1 /(20 A)$ has to be chosen.) The inequality (3.36) now gives

$$
\int_{G} f_{+}^{2} g \geq \frac{1}{2} \int_{D_{1} \cup D_{2}} f_{+}^{2} g \geq \frac{1}{80} .
$$

Now, if there exists an $x_{2} \in[0,1 / 4]$ such that for all $x_{1} \in[0,1 / 8], f_{+}\left(x_{1}, x_{2}\right)>$ $A \eta$ (recall that $\eta=1 / \sqrt{2}$ ), then we may apply Lemma 3.12 to obtain the estimate

$$
\int_{\tilde{D}}|\nabla f|^{2} g \geq \min \left(\frac{(1-\eta)^{2}}{4 \eta^{2} L^{2}}, \frac{4(1-\eta)^{2}}{\eta^{2} C_{H} L}\right) \geq \min \left(\frac{16}{10^{2} L^{2}}, \frac{64}{10^{2} C_{H} L}\right) .
$$

This implies (3.32). Here we used the inequality $(1-\eta)^{2} \geq 8 / 10^{2},(\eta=$ $1 / \sqrt{2})$.

Thus we may assume that for all $x_{2} \in[0,1 / 4]$ there is an $x_{1} \in[0,1 / 8]$ such that $f_{+}\left(x_{1}, x_{2}\right) \leq A \eta$. Let

$$
h\left(x_{2}\right)=\sup \left\{x_{1} \in[0,1 / 8]: f\left(x_{1}, x_{2}\right) \leq A \eta\right\} .
$$


Recall that $p\left(x_{2}\right)$ is such that $\left(p\left(x_{2}\right), x_{2}\right) \in \partial D$. For $x_{2} \in[0,1 / 4]$ let

$$
G_{x_{2}}^{\prime}=\left\{x_{1} \in\left[h\left(x_{2}\right), p\left(x_{2}\right)\right):\left(x_{1}, x_{2}\right) \in G\right\}
$$

and

$$
G_{x_{2}}^{\prime \prime}=\left\{x_{1} \in\left[0, h\left(x_{2}\right)\right):\left(x_{1}, x_{2}\right) \in G\right\} .
$$

By Lemma 3.4 for $x_{2} \in[0,1 / 4]$ we have

$$
\begin{aligned}
& \int_{h\left(x_{2}\right)}^{p\left(x_{2}\right)}\left(\frac{\partial f_{+}}{\partial x_{1}}\right)^{2}\left(x_{1}, x_{2}\right) g\left(x_{1}, x_{2}\right) d x_{1} \\
& \geq \frac{(1-\eta)^{2}}{\left|p\left(x_{2}\right)-h\left(x_{2}\right)\right|^{2}} \int_{G_{x_{2}}^{\prime}} f_{+}^{2}\left(x_{1}, x_{2}\right) g\left(x_{1}, x_{2}\right) d x_{1} .
\end{aligned}
$$

By the Harnack inequality (3.12) and Lemma 3.5 for $x_{2} \in[0,1 / 4]$ we have

$$
\begin{aligned}
& \int_{0}^{h\left(x_{2}\right)}\left(\frac{\partial f_{+}}{\partial x_{1}}\right)^{2}\left(x_{1}, x_{2}\right) g\left(x_{1}, x_{2}\right) d x_{1} \\
\geq & \frac{g\left(0, x_{2}\right)}{C_{H}} \int_{0}^{h\left(x_{2}\right)}\left(\frac{\partial f_{+}}{\partial x_{1}}\right)^{2}\left(x_{1}, x_{2}\right) d x_{1} \\
\geq & \frac{g\left(0, x_{2}\right)(1-\eta)^{2}}{C_{H} h^{2}\left(x_{2}\right)} \int_{G_{x_{2}}^{\prime \prime}} f_{+}^{2}\left(x_{1}, x_{2}\right) d x_{1} \\
\geq & \frac{64(1-\eta)^{2}}{C_{H}} \int_{G_{x_{2}}^{\prime \prime}} f_{+}^{2}\left(x_{1}, x_{2}\right) g\left(x_{1}, x_{2}\right) d x_{1} .
\end{aligned}
$$

It follows that

$$
\begin{aligned}
& \int_{\tilde{D}}|\nabla f|^{2} g \geq \int_{D_{1} \cup D_{2}}\left(\frac{\partial f}{\partial x_{1}}\right)^{2} g \\
\geq & \int_{0}^{1 / 4} \int_{h\left(x_{2}\right)}^{p\left(x_{2}\right)}\left(\frac{\partial f_{+}}{\partial x_{1}}\right)^{2}\left(x_{1}, x_{2}\right) g\left(x_{1}, x_{2}\right) d x_{1} d x_{2} \\
+ & \int_{0}^{1 / 4} \int_{0}^{h\left(x_{2}\right)}\left(\frac{\partial f_{+}}{\partial x_{1}}\right)^{2}\left(x_{1}, x_{2}\right) g\left(x_{1}, x_{2}\right) d x_{1} d x_{2} \\
\geq & \min \left(1, \frac{64}{C_{H}}\right) \frac{(1-\eta)^{2}}{L^{2}} \int_{0}^{1 / 4} \int_{G_{x_{2}}^{\prime} \cup G_{x_{2}}^{\prime \prime}} f_{+}^{2}\left(x_{1}, x_{2}\right) g\left(x_{1}, x_{2}\right) d x_{1} d x_{2} \\
= & \min \left(1, \frac{64}{C_{H}}\right) \frac{(1-\eta)^{2}}{L^{2}} \int_{G} f_{+}^{2} g \\
\geq & \min \left(\frac{1}{10^{3} L^{2}}, \frac{64}{10^{3} C_{H} L^{2}}\right),
\end{aligned}
$$


which completes Case 2. Here we used the fact that $\int_{G} f_{+}^{2} g \geq 1 / 80$ and $(1-\eta)^{2} \geq 8 / 10^{2}$.

Case 3. For this case we use Lemmas 3.9 and 3.10. We have $\int_{\tilde{D}} f_{+}^{2} g \geq m$ and $\int_{\tilde{D}} f_{-}^{2} g \geq m$. Put $\delta_{1}=\sqrt{4 / 10}, \delta_{2}=\sqrt{4 / 10}$. Then we have $(1-$ $\left.\sqrt{\delta_{1}}\right)^{2}\left(1-\delta_{2}\right) m \geq 4 \cdot 10^{-5}$. If

$$
\int_{D_{1} \cup D_{2}} f_{+}^{2} g<m / 10 \quad \text { or } \quad \int_{D_{1} \cup D_{2}} f_{-}^{2} g<m / 10
$$

then (3.33) follows from Lemma 3.9. If

$$
\int_{D_{1} \cup D_{3}} f_{+}^{2} g<m / 10 \text { or } \int_{D_{1} \cup D_{3}} f_{-}^{2} g<m / 10
$$

then (3.33) follows from Lemma 3.10.

Case 4. Set

$$
a_{1}=\inf \left\{x_{1} \in[0, L / 4]: \forall x_{2} \in[0,1 / 4]\left|f\left(x_{1}, x_{2}\right)\right|>0\right\} .
$$

We have $a_{1} \in[0, L / 4]$ and for all $x_{2} \in[0,1 / 4], f\left(a_{1}, x_{2}\right) \geq 0$ or for all $x_{2} \in[0,1 / 4], f\left(a_{1}, x_{2}\right) \leq 0$. We may assume that for all $x_{2} \in[0,1 / 4]$, $f\left(a_{1}, x_{2}\right) \geq 0$. We have

$$
\begin{aligned}
\int_{D_{1} \cup D_{2}}|\nabla f|^{2} g & \geq \int_{0}^{1 / 4} \int_{0}^{a_{1}}\left(\frac{\partial f}{\partial x_{2}}\right)^{2} g d x_{1} d x_{2} \\
& +\int_{0}^{1 / 4} \int_{a_{1}}^{p\left(x_{2}\right)}\left(\frac{\partial f}{\partial x_{1}}\right)^{2} g d x_{1} d x_{2} .
\end{aligned}
$$

Note that for all $x_{1} \in\left[0, a_{1}\right)$ there exists $x_{2} \in[0,1 / 4]$ such that $f\left(x_{1}, x_{2}\right)=0$. For $x_{1} \in\left[0, a_{1}\right)$, set

$$
z\left(x_{1}\right)=\sup \left\{x_{2} \in[0,1 / 4]: f\left(x_{1}, x_{2}\right)=0\right\} .
$$

By Lemma 3.2 we obtain

$$
\int_{0}^{a_{1}} \int_{z\left(x_{1}\right)}^{1 / 4}\left(\frac{\partial f}{\partial x_{2}}\right)^{2} g d x_{2} d x_{1} \geq \int_{0}^{a_{1}} \frac{1}{\left((1 / 4)-z\left(x_{1}\right)\right)^{2}} \int_{z\left(x_{1}\right)}^{1 / 4} f^{2} g d x_{2} d x_{1} .
$$


By the Harnack inequality (3.11) and Lemma 3.3,

$$
\begin{aligned}
\int_{0}^{a_{1}} \int_{0}^{z\left(x_{1}\right)}\left(\frac{\partial f}{\partial x_{2}}\right)^{2} g d x_{2} d x_{1} & \geq \frac{g\left(x_{1}, 0\right)}{C_{H}} \int_{0}^{a_{1}} \int_{0}^{z\left(x_{1}\right)}\left(\frac{\partial f}{\partial x_{2}}\right)^{2} d x_{2} d x_{1} \\
& \geq \frac{g\left(x_{1}, 0\right)}{C_{H} z^{2}\left(x_{1}\right)} \int_{0}^{a_{1}} \int_{0}^{z\left(x_{1}\right)} f^{2} d x_{2} d x_{1} \\
& \geq \frac{1}{C_{H} z^{2}\left(x_{1}\right)} \int_{0}^{a_{1}} \int_{0}^{z\left(x_{1}\right)} f^{2}(x) g d x_{2} d x_{1} .
\end{aligned}
$$

This and (3.40) give

$$
\int_{0}^{1 / 4} \int_{0}^{a_{1}}\left(\frac{\partial f}{\partial x_{2}}\right)^{2} g d x_{1} d x_{2} \geq \frac{16}{C_{H}} \int_{0}^{1 / 4} \int_{0}^{a_{1}} f^{2} g d x_{1} d x_{2} .
$$

Next, we estimate the integral in (3.39). Recall that for all $x_{2} \in[0,1 / 4]$, $f\left(a_{1}, x_{2}\right) \geq 0$. It follows that $f_{-}\left(a_{1}, x_{2}\right)=0$. From Lemma 3.2 we obtain

$$
\int_{0}^{1 / 4} \int_{a_{1}}^{p\left(x_{2}\right)}\left(\frac{\partial f_{-}}{\partial x_{1}}\right)^{2} g d x_{1} d x_{2} \geq \int_{0}^{1 / 4} \frac{1}{\left(p\left(x_{2}\right)-a_{1}\right)^{2}} \int_{a_{1}}^{p\left(x_{2}\right)} f_{-}^{2} g d x_{1} d x_{2} .
$$

By this, (3.43) and (3.38) we finally obtain

$$
\begin{aligned}
\int_{D_{1} \cup D_{2}}|\nabla f|^{2} g & \geq \min \left(1, \frac{16}{C_{H}}\right) \frac{1}{L^{2}} \int_{D_{1} \cup D_{2}} f_{-}^{2} g \geq \min \left(1, \frac{16}{C_{H}}\right) \frac{m}{10} \\
& =\min \left(\frac{1}{32 \cdot 10^{2}}, \frac{1}{2 \cdot 10^{2} C_{H}}\right) \frac{1}{L^{2}},
\end{aligned}
$$

which completes Case 4.

Case 5. For $x_{1} \in[0, L / 4)$, set

$$
z\left(x_{1}\right)=\sup \left\{x_{2} \in[0,1 / 4]: f\left(x_{1}, x_{2}\right)=0\right\} .
$$

By Lemma 3.2 we have

$$
\begin{aligned}
& \int_{0}^{L / 4} \int_{z\left(x_{1}\right)}^{r\left(x_{1}\right)}\left(\frac{\partial f}{\partial x_{2}}\right)^{2} g d x_{2} d x_{1} \\
& \geq \int_{0}^{L / 4} \frac{1}{\left(r\left(x_{1}\right)-z\left(x_{1}\right)\right)^{2}} \int_{z\left(x_{1}\right)}^{r\left(x_{1}\right)} f^{2} g d x_{2} d x_{1} .
\end{aligned}
$$

Recall that $r:(-L, L) \rightarrow(0,1]$ is such that $\left(x_{1}, r\left(x_{1}\right)\right) \in \partial D$. 
By the Harnack inequality (3.11) and Lemma 3.3 (exactly as in (3.41)(3.42)) we have

$$
\int_{0}^{L / 4} \int_{0}^{z\left(x_{1}\right)}\left(\frac{\partial f}{\partial x_{2}}\right)^{2} g d x_{2} d x_{1} \geq \int_{0}^{L / 4} \frac{1}{C_{H} z^{2}\left(x_{1}\right)} \int_{0}^{z\left(x_{1}\right)} f^{2} g d x_{2} d x_{1} .
$$

This and (3.44) give

$$
\int_{D_{1} \cup D_{3}}|\nabla f|^{2} g \geq \min \left(1, \frac{16}{C_{H}}\right) \int_{D_{1} \cup D_{3}} f^{2} g \geq \min \left(\frac{1}{32 \cdot 10^{2}}, \frac{1}{2 \cdot 10^{2} C_{H}}\right) \frac{1}{L^{2}},
$$

which completes Case 5 and hence the proof of Theorem 3.1

We now present the example mentioned above which shows that the assumption of Theorem 3.1 that $g$ satisfies the Harnack inequality is necessary. We will show that without it there does not exist a universal constant $C_{P}>0$ such that (3.1) holds.

Example 3.1. Let $\varepsilon \in(0,1 / 4)$. Let $f_{1}:[0,1] \rightarrow \mathbb{R}$ be defined by

$$
f_{1}(x)=\left\{\begin{aligned}
-\varepsilon, & \text { for } \quad x \in\left[0, x_{1}\right) \\
c_{1}\left(x-x_{1}\right)^{2}-\varepsilon, & \text { for } \quad x \in\left[x_{1}, \varepsilon\right) \\
\frac{1}{\varepsilon^{2}}\left(x^{(-\ln 2 / \ln \varepsilon)}-\frac{1}{2}\right), & \text { for } \quad x \in[\varepsilon, 1]
\end{aligned}\right.
$$

Note that $-\ln 2 / \ln \varepsilon=\log _{\varepsilon}(1 / 2)$, so $f_{1}(\varepsilon)=0$. We choose $x_{1}$ and $c_{1}$ so that $f_{1} \in C^{1}[0,1]$. That is, $c_{1}\left(\varepsilon-x_{1}\right)^{2}-\varepsilon=0$ and

$$
\left[\left(c_{1}\left(x-x_{1}\right)^{2}-\varepsilon\right)^{\prime}\right]_{x=\varepsilon}=\left[\frac{1}{\varepsilon^{2}}\left(x^{(-\ln 2 / \ln \varepsilon)}-\frac{1}{2}\right)^{\prime}\right]_{x=\varepsilon} .
$$

Hence, $c_{1}, x_{1}$ satisfy

$$
c_{1}\left(\varepsilon-x_{1}\right)^{2}=\varepsilon, \quad 2 c_{1}\left(\varepsilon-x_{1}\right)=\frac{-\ln 2}{2 \varepsilon^{3} \ln \varepsilon} .
$$

This gives

$$
x_{1}=\varepsilon-\frac{4 \varepsilon^{4} \ln \varepsilon}{-\ln 2}, \quad c_{1}=\frac{\ln ^{2} 2}{16 \varepsilon^{7} \ln ^{2} \varepsilon} .
$$

For $\varepsilon \in(0,1 / 4)$ we have

$$
0<4 \varepsilon^{4} \ln \varepsilon /(-\ln 2)<8 \varepsilon^{4}|\ln \varepsilon| \leq 8 \varepsilon^{3} \leq \varepsilon / 2,
$$

hence $x_{1} \in(\varepsilon / 2, \varepsilon)$. 
Now let $g_{1}:[0,1] \rightarrow(0, \infty)$ be defined by

$$
g_{1}(x)=\left\{\begin{aligned}
\frac{c_{2}}{\varepsilon}, & \text { for } \quad x \in[0, \varepsilon / 4), \\
c_{3}\left(x-\frac{\varepsilon}{4}\right)+\frac{c_{2}}{\varepsilon}, & \text { for } x \in[\varepsilon / 4, \varepsilon / 2), \\
\varepsilon^{4}, & \text { for } x \in[\varepsilon / 2,1] .
\end{aligned}\right.
$$

We choose $c_{3}$ so that $g \in C[0,1]$. That is, $c_{3}(\varepsilon / 2-\varepsilon / 4)+c_{2} / \varepsilon=\varepsilon^{4}$ which gives $c_{3}=4 \varepsilon^{3}-4 c_{2} / \varepsilon^{2}$. The constant $c_{2}$ will be chosen later.

Let $D=B(0,1) \subset \mathbb{R}^{2}$ and for $x \in B(0,1)$, let $f(x)=f_{1}(|x|), g(x)=$ $g_{1}(|x|)$. We choose $c_{2}>0$ so that

$$
\int_{B(0,1)} f(x) g(x) d x=0 .
$$

We will show that $c_{2} / \varepsilon \geq \varepsilon^{4}$ for $\varepsilon \in(0,1 / 4)$ and so $g$ satisfies the assumption (iii) of Theorem 3.1. Observe first that for $\varepsilon \in(0,1 / 4)$ we have $0<-\ln 2 / \ln \varepsilon \leq 1 / 2$. Thus for $\rho \geq 1 / 2$ we have $\rho^{(-\ln 2 / \ln \varepsilon)} \geq 1 / \sqrt{2}$. It follows that

$$
\begin{aligned}
\int_{B(0,1) \backslash B(0, \varepsilon)} f g>\int_{B(0,1) \backslash B(0,1 / 2)} f g & =2 \pi \varepsilon^{2} \int_{1 / 2}^{1}\left(\rho^{(-\ln 2 / \ln \varepsilon)}-\frac{1}{2}\right) \rho d \rho \\
& \geq \frac{\pi \varepsilon^{2}(\sqrt{2}-1)}{4} .
\end{aligned}
$$

If $c_{2} / \varepsilon<\varepsilon^{4}$, then we would have

$$
\int_{B(0, \varepsilon)} f g>-2 \pi \int_{0}^{\varepsilon} \varepsilon \varepsilon^{4} \rho d \rho=-\pi \varepsilon^{7} \geq-\pi \varepsilon^{2}(1 / 4)^{5} .
$$

Thus

$$
\int_{B(0,1)} f g>0
$$

which gives a contradiction. Hence $c_{2} / \varepsilon \geq \varepsilon^{4}$. (In fact, it is not difficult to see that for any $\varepsilon \in(0,1 / 4), c_{2} \in(3 / 10,8)$. )

Now, it is easy to see that for an arbitrary $\varepsilon \in(0,1 / 4)$, the functions $f$, $g$ satisfy all the assumptions of Theorem 3.1 except the assumption on the Harnack inequality for $g$.

Since $\rho^{(-\ln 2 / \ln \varepsilon)} \geq 1 / \sqrt{2}$ for $\rho \geq 1 / 2$ and $\varepsilon \in(0,1 / 4)$, we obtain that

$$
\begin{aligned}
\int_{B(0,1)} f^{2} g & \geq \int_{B(0,1) \backslash B(0,1 / 2)} f^{2} g \\
& =2 \pi \int_{1 / 2}^{1}\left(\rho^{(-\ln 2 / \ln \varepsilon)}-\frac{1}{2}\right)^{2} \rho d \rho \\
& \geq \frac{\pi(\sqrt{2}-1)^{2}}{8} .
\end{aligned}
$$


Also, for any $x \in B(0,1)$ we have

$$
|\nabla f(x)|^{2}=\left(\frac{\partial f}{\partial x_{1}}(x)\right)^{2}+\left(\frac{\partial f}{\partial x_{2}}(x)\right)^{2}=\left(f_{1}^{\prime}(|x|)\right)^{2} .
$$

It follows that

$$
\begin{aligned}
\int_{B(0,1) \backslash B(0, \varepsilon)}|\nabla f(x)|^{2} g(x) d x & =2 \pi \int_{\varepsilon}^{1}\left|f_{1}^{\prime}(\rho)\right|^{2} g(\rho) \rho d \rho \\
& =\frac{2 \pi \ln ^{2} 2}{\ln ^{2} \varepsilon} \int_{\varepsilon}^{1} \rho^{(-2 \ln 2 / \ln \varepsilon)-1} d \rho \\
& =\frac{-\pi \ln 2}{\ln \varepsilon}\left[\rho^{-2 \ln 2 / \ln \varepsilon}\right]_{\varepsilon}^{1} \leq \frac{\pi \ln 2}{|\ln \varepsilon|} .
\end{aligned}
$$

We have $x_{1} \geq \varepsilon / 2$ so $g(x)=\varepsilon^{4}$ for $x \in B(0, \varepsilon) \backslash B\left(0, x_{1}\right)$. Hence,

$$
\begin{aligned}
\int_{B(0, \varepsilon) \backslash B\left(0, x_{1}\right)}|\nabla f(x)|^{2} g(x) d x & =2 \pi \int_{x_{1}}^{\varepsilon}\left|f_{1}^{\prime}(\rho)\right|^{2} g(\rho) \rho d \rho \\
& \leq 2 \pi \int_{x_{1}}^{\varepsilon} 4 c_{1}^{2}\left(\rho-x_{1}\right)^{2} \varepsilon^{5} d \rho=\frac{\varepsilon^{3} 2 \pi \ln 2}{3|\ln \varepsilon|} .
\end{aligned}
$$

We also have

$$
\int_{B\left(0, x_{1}\right)}|\nabla f|^{2} g=0
$$

Finally we obtain that $\int_{B(0,1)}|\nabla f|^{2} g \rightarrow 0$, when $\varepsilon \rightarrow 0$. On the other hand, for any $\varepsilon \in(0,1 / 4)$ we have $\int_{B(0,1)} f^{2} g \geq \pi(\sqrt{2}-1)^{2} / 8>0$.

This means that without the assumption (iv) there does not exist a universal constant $C_{P}$ such that (3.1) holds and completes our example.

\section{The Spectral Gap Estimate, Proof of Theorem 1.1}

In this section we prove our estimate for $\lambda_{2}-\lambda_{1}$. This will be done by exploiting the connection between the eigenvalue problem for the Cauchy process and the eigenvalue problem for the mixed Steklov eigenvalue problem which was established in [5]. We first recall some notation. Let $D \subset \mathbb{R}^{2}$ be as in Theorem 1.1. As before, $\left\{T_{t}^{D}\right\}_{t \geq 0}$ is the semigroup of the Cauchy process killed upon exiting $D$ with corresponding eigenvalues $\left\{\lambda_{n}\right\}_{n=1}^{\infty}$ and eigenfunctions $\left\{\varphi_{n}\right\}_{n=1}^{\infty}$. The functions $\varphi_{n}$ are defined on $D$ but we extend 
them to all of $\mathbb{R}^{2}$ by setting them to be zero on $D^{c}$. Recall that $\varphi_{n}$ is continuous on $\mathbb{R}^{2}$ (see inequality (3.2) in [5]). In fact, these functions are real analytic in $D$, as shown in [5]. Since $D$ is bounded it follows that $\varphi_{n} \in L^{1}\left(\mathbb{R}^{2}\right)$.

In this section we will simply write $p(t, x, y)=p^{(1)}(t, x, y)$ for the Cauchy density in $\mathbb{R}^{2}$. With this notation we have as in (1.2) that

$$
p(t, x, y)=\frac{t}{2 \pi\left(t^{2}+|x-y|^{2}\right)^{3 / 2}}, \quad t>0, x, y \in \mathbb{R}^{2} .
$$

We put

$$
T_{t} f(x)=\int_{\mathbb{R}^{2}} p(t, x, y) f(y) d y, \quad f \in L^{1}\left(\mathbb{R}^{2}\right) .
$$

Let $H=\left\{(x, t): x \in \mathbb{R}^{2}, t \geq 0\right\}, H_{+}=\left\{(x, t): x \in \mathbb{R}^{2}, t>0\right\}$ and for $(x, t) \in H_{+}$set $u_{n}(x, t)=T_{t} \varphi_{n}(x)$. Since $\varphi_{n}$ are continuous on $\mathbb{R}^{2}, u_{n}$ are continuous on $H$. As shown in [5], Theorem 1.1, the functions $u_{n}$ are eigenvalue functions for mixed Steklov eigenvalue problem:

$$
\begin{aligned}
& \Delta u_{n}(x, t)=0, \quad(x, t) \in H_{+}, \\
& \frac{\partial u_{n}}{\partial t}(x, t)=-\lambda_{n} u_{n}(x, 0), \quad x \in D, \\
& u_{n}(x, 0)=0 \quad x \in D^{c},
\end{aligned}
$$

where

$$
\Delta=\frac{\partial^{2}}{\partial x_{1}^{2}}+\frac{\partial^{2}}{\partial x_{2}^{2}}+\frac{\partial^{2}}{\partial t^{2}}
$$

This connection between the eigenvalues of the Cauchy process holds not only on $\mathbb{R}^{2}$ but in all dimensions and for a wider class of domains, as shown in [5]. The important point for us here is that by Theorem 2.1 in [6],

$$
\lambda_{2}-\lambda_{1}=\int_{H}\left|\nabla\left(\frac{u_{2}}{u_{1}}\right)(x, t)\right|^{2} u_{1}^{2}(x, t) d x d t .
$$

This formula is the starting point for our estimates of $\lambda_{2}-\lambda_{1}$. Since we will now have functions defined in both $\mathbb{R}^{2}$ and $H$, we will write

$$
\nabla=\left(\frac{\partial}{\partial x_{1}}, \frac{\partial}{\partial x_{2}}, \frac{\partial}{\partial t}\right)
$$

for the gradient of functions in $H$ and

$$
\nabla_{x}=\left(\frac{\partial}{\partial x_{1}}, \frac{\partial}{\partial x_{2}}\right)
$$


for the gradient of functions in $\mathbb{R}^{2}$.

We start by recalling the following lemma from [6] (Lemma 2.1).

Lemma 4.1. For any $x \in D$ and $t>0$ we have

$$
u_{1}(x, t) \geq e^{-\lambda_{1} t} \varphi_{1}(x) .
$$

An immediate conclusion of (4.4) and Lemma 4.1 is

Lemma 4.2. For any $T>0 w$ have

$$
\lambda_{2}-\lambda_{1} \geq e^{-2 \lambda_{1} T} \int_{0}^{T} \int_{D}\left|\nabla\left(\frac{u_{2}}{u_{1}}\right)(x, t)\right|^{2} \varphi_{1}^{2}(x) d x d t .
$$

Lemma 4.3. Let $D$ be as in Theorem 1.1. Assume that $f: D \rightarrow \mathbb{R}$, $f \in C^{1}(D)$ and that $f$ is bounded and symmetric with respect to both axes and

$$
\int_{D} f(x) \varphi_{1}^{2}(x) d x=0
$$

Then

$$
\int_{D}\left|\nabla_{x} f(x)\right|^{2} \varphi_{1}^{2}(x) d x \geq \frac{C_{\varphi}}{L^{2}} \int_{D} f^{2}(x) \varphi_{1}^{2}(x) d x
$$

where $C_{\varphi}=4 \cdot 10^{-6}$.

Proof. We apply Theorem 3.1 to $g=\varphi_{1}^{2}$. The function $\varphi_{1}^{2}$ satisfies the assumptions of Theorem 3.1 by Theorem 2.1 and Corollary 2.1. Corollary 2.1 gives that $g=\varphi_{1}^{2}$ satisfies the Harnack inequality with the constant $C_{H}=5 \cdot 10^{3}$. Substituting $C_{H}=5 \cdot 10^{3}$ in (3.2) we get

$$
C_{P}=\min \left(\frac{16}{10^{5}}, \frac{2}{10^{2} \cdot 5 \cdot 10^{3}}\right)=\frac{4}{10^{6}},
$$

which gives the constant above.

Corollary 4.1. Let $D$ be as in Theorem 1.1. Assume that $f: D \rightarrow \mathbb{R}$, $f \in C^{1}(D)$ and that $f$ is bounded and symmetric with respect to both axes. Then

$$
\int_{D}\left|\nabla_{x} f(x)\right|^{2} \varphi_{1}^{2}(x) d x \geq \frac{C_{\varphi}}{L^{2}}\left(\int_{D} f^{2}(x) \varphi_{1}^{2}(x) d x-\left(\int_{D} f(x) \varphi_{1}^{2}(x) d x\right)^{2}\right)
$$

where $C_{\varphi}$ is the same constant as in Lemma 4.3. 
Proof. Simply apply the Lemma 4.3 to the function

$$
\tilde{f}(x)=f(x)-\int_{D} f(y) \varphi_{1}^{2}(y) d y .
$$

Corollary 4.2. Let $D$ be as in Theorem 1.1. Assume that $u: D \times[0,1] \rightarrow \mathbb{R}$ is such that for any fixed $t \in(0,1]$ the function $u(\cdot, t) \in C^{1}(D)$ is bounded and symmetric with respect to both axes. Then for any $t \in(0,1]$,

$$
\begin{aligned}
& \int_{D}\left|\nabla_{x} u(x, t)\right|^{2} \varphi_{1}^{2}(x) d x \\
& \geq \frac{C_{\varphi}}{L^{2}}\left(\int_{D} u^{2}(x, t) \varphi_{1}^{2}(x) d x-\left(\int_{D} u(x, t) \varphi_{1}^{2}(x) d x\right)^{2}\right)
\end{aligned}
$$

where $C_{\varphi}$ is the same constant as in Lemma 4.3.

Lemma 4.4. Let $D$ be as in Theorem 1.1. Let $u: D \times[0,1] \rightarrow \mathbb{R}$ be continuous bounded and such that for any fixed $x \in D, u(x, \cdot) \in C^{1}[0,1]$. We also assume that

$$
\int_{D} u(x, 0) \varphi_{1}^{2}(x) d x=0
$$

Then for any $t \in(0,1]$,

$$
\int_{0}^{t} \int_{D}\left(\frac{\partial u}{\partial s}(x, s)\right)^{2} \varphi_{1}^{2}(x) d x d s \geq\left(\int_{D} u(x, t) \varphi_{1}^{2}(x) d x\right)^{2} .
$$

Proof. Let $t \in(0,1]$. By (4.5),

$$
\begin{aligned}
\left(\int_{D} u(x, t) \varphi_{1}^{2}(x) d x\right)^{2} & =\left(\int_{D}(u(x, t)-u(x, 0)) \varphi_{1}^{2}(x) d x\right)^{2} \\
& =\left(\int_{D} \int_{0}^{t} \frac{\partial u}{\partial s}(x, s) d s \varphi_{1}^{2}(x) d x\right)^{2} \\
& \leq\left(\int_{0}^{t} \int_{D}\left|\frac{\partial u}{\partial s}(x, s)\right| \varphi_{1}^{2}(x) d x d s\right)^{2} .
\end{aligned}
$$

By Schwarz inequality this is bounded above by

$$
\int_{0}^{t} d s \int_{0}^{t}\left(\int_{D}\left|\frac{\partial u}{\partial s}(x, s)\right| \varphi_{1}^{2}(x) d x\right)^{2} d s .
$$


Using our assumption that $t \leq 1$, and again Schwarz inequality, the previous quantity is bounded above by

$$
\int_{0}^{t} \int_{D} \varphi_{1}^{2}(x) d x \int_{D}\left(\frac{\partial u}{\partial s}(x, s)\right)^{2} \varphi_{1}^{2}(x) d x d s .
$$

Lemma 4.5. Let $D$ be as in Theorem 1.1. Let $u: D \times[0,1] \rightarrow \mathbb{R}$ be continuous, bounded and such that for any fixed $x \in D, u(x, \cdot) \in C^{1}[0,1]$ and for any fixed $t \in(0,1], u(\cdot, t) \in C^{1}(D)$ is symmetric with respect to both axes. We also assume that

$$
\int_{D} u(x, 0) \varphi_{1}^{2}(x) d x=0 .
$$

Then for any $0<T \leq 1$,

$$
\begin{aligned}
& \int_{0}^{T} \int_{D}|\nabla u(x, t)|^{2} \varphi_{1}^{2}(x) d x d t \\
& \geq \frac{C_{\varphi}}{L^{2}} \int_{0}^{T} \int_{D}\left(u^{2}(x, t)+\left(\frac{\partial u}{\partial t}(x, t)\right)^{2}\right) \varphi_{1}^{2}(x) d x d t
\end{aligned}
$$

where $C_{\varphi}$ is the same constant as in Lemma 4.3 .

Proof. Let $0<T \leq 1$. By Corollary 4.2 and Lemma 4.4 we have

$$
\begin{aligned}
& \int_{0}^{T} \int_{D}\left|\nabla_{x} u(x, t)\right|^{2} \varphi_{1}^{2}(x) d x d t \\
& \geq \frac{C_{\varphi}}{L^{2}}\left(\int_{0}^{T} \int_{D} u^{2}(x, t) \varphi_{1}^{2}(x) d x d t-\int_{0}^{T} \int_{0}^{t} \int_{D}\left(\frac{\partial u}{\partial s}(x, s)\right)^{2} \varphi_{1}^{2}(x) d x d s d t\right) \\
& \geq \frac{C_{\varphi}}{L^{2}}\left(\int_{0}^{T} \int_{D} u^{2}(x, t) \varphi_{1}^{2}(x) d x d t-\int_{0}^{T} \int_{D}\left(\frac{\partial u}{\partial t}(x, t)\right)^{2} \varphi_{1}^{2}(x) d x d t\right) .
\end{aligned}
$$

It follows from this that

$$
\begin{aligned}
& \int_{0}^{T} \int_{D}|\nabla u(x, t)|^{2} \varphi_{1}^{2}(x) d x d t \\
& =\int_{0}^{T} \int_{D}\left(\left|\nabla_{x} u(x, t)\right|^{2}+\left(\frac{\partial u}{\partial t}(x, t)\right)^{2}\right) \varphi_{1}^{2}(x) d x d t \\
& \geq \frac{C_{\varphi}}{L^{2}} \int_{0}^{T} \int_{D} u^{2}(x, t) \varphi_{1}^{2}(x) d x d t-\frac{C_{\varphi}}{L^{2}} \int_{0}^{T} \int_{D}\left(\frac{\partial u}{\partial t}(x, t)\right)^{2} \varphi_{1}^{2}(x) d x d t \\
& +\int_{0}^{T} \int_{D}\left(\frac{\partial u}{\partial t}(x, t)\right)^{2} \varphi_{1}^{2}(x) d x d t
\end{aligned}
$$


which gives the assertion of the lemma.

Lemma 4.6. Let $f \in C^{1}[0,1]$. Then for any $0<T \leq 1$,

$$
\int_{0}^{T} f^{2}(t)+\left(f^{\prime}(t)\right)^{2} d t \geq \frac{T f^{2}(0)}{2} .
$$

Proof. Let $0<T \leq 1$. Let $a \in[0, T]$ be such that $f(a)=\min _{t \in[0, T]} f(t)$. Then,

$$
\begin{aligned}
\int_{0}^{T} f^{2}(t)+\left(f^{\prime}(t)\right)^{2} d t & \geq T f^{2}(a)+T^{-1}\left(\int_{0}^{a} f^{\prime}(t) d t\right)^{2} \\
& \geq T\left(f^{2}(a)+(f(a)-f(0))^{2}\right) \\
& \geq T f^{2}(0) / 2
\end{aligned}
$$

Proof of Theorem 1.1. Recall that $D$ is symmetric with respect to both coordinate axes. For $x=\left(x_{1}, x_{2}\right)$, set $\widehat{x}=\left(-x_{1}, x_{2}\right)$. Theorem 4.3 in [5] gives that the existence of an eigenfunction $\varphi_{*}$ with eigenvalue $\lambda_{*}$ which is antisymmetric relative to the $x_{1}$-axis $\left(\varphi_{*}(x)=-\varphi_{*}(\widehat{x}), x \in D\right.$ ) and (up to a sign) $\varphi_{*}(x)>0$ for $x \in D_{+}$and $\varphi_{*}(x)<0$ for $x \in D_{-}$. Moreover, if $\varphi$ is any eigenfunction with eigenvalue $\lambda$ such that $\varphi$ is antisymmetric relative to the $x_{1}$-axis and $\varphi$ is different from $\varphi_{*}\left(\varphi \notin \operatorname{Span}\left\{\varphi_{*}\right\}\right)$, then $\lambda_{*}<\lambda$. In other words, $\varphi_{*}$ has the smallest eigenvalue amongst all eigenfunctions which are antisymmetric relative to the $x_{1}$-axis. Therefore, if there exists an eigenfunction corresponding to $\lambda_{2}$ which is antisymmetric with respect to the $x_{1}$-axis, then $\lambda_{2}=\lambda_{*}$. Hence if $\lambda_{2}=\lambda_{*}$ it follows from Corollary 1.1 of [6] that

$$
\lambda_{2}-\lambda_{1}=\lambda_{*}-\lambda_{1} \geq \min \left(\frac{C_{2} r_{D}}{L^{2}}, \frac{C_{2}^{\prime}}{r_{D}}\right),
$$

where $C_{2}=\left(3 \pi^{2} /(16 \pi+12)\right) \geq 4 / 10, C_{2}^{\prime}=\left(4 C_{2}\right) / \pi^{2} \geq 1 / 6$ and $r_{D}$ is the inradius of $D$ as defined in the introduction. By our assumptions on $D$ we have $1 \geq r_{D} \geq \sqrt{2} / 2$. It follows that

$$
\lambda_{2}-\lambda_{1} \geq \min \left(\frac{\sqrt{2}}{5 L^{2}}, \frac{1}{6}\right) \geq \frac{1}{6 L^{2}} .
$$

This implies (1.5) (the assertion of Theorem 1.1). 
Similarly, if there exists an eigenfunction corresponding to $\lambda_{2}$ which is antisymmetric with respect to the $x_{2}$-axis, then Corollary 1.1 from [6] (applied for $x_{2}$-axis) gives

$$
\lambda_{2}-\lambda_{1} \geq \min \left(\frac{C_{2} r_{D}}{1^{2}}, \frac{C_{2}^{\prime}}{r_{D}}\right) \geq \min \left(\frac{\sqrt{2}}{5}, \frac{1}{6}\right) \geq \frac{1}{6},
$$

which again implies (1.5).

Thus we may assume that there is no eigenfunction corresponding to $\lambda_{2}$ which is antisymmetric with respect to the $x_{1}$-axis or the $x_{2}$-axis. We will show that under these assumption there is an eigenfunction corresponding to $\lambda_{2}$ which is symmetric with respect to both the $x_{1}$-axis and the $x_{2}$-axis. Toward this end, let $\psi$ be an eigenfunction corresponding to $\lambda_{2}$. Put $\widehat{\psi}(x)=$ $\psi(\widehat{x})$. We have

$$
T_{t}^{D} \widehat{\psi}(x)=\int_{D} p_{D}(t, x, y) \psi(\widehat{y}) d y=\int_{D} p_{D}(t, x, \widehat{z}) \psi(z) d z,
$$

where the last equality follows by the substitution $z=\widehat{y}$. By Lemma 4.6 in [5] this last quantity equals

$$
\int_{D} p_{D}(t, \widehat{x}, z) \psi(z) d z=e^{-\lambda_{2} t} \psi(\widehat{x})=e^{-\lambda_{2} t} \widehat{\psi}(x) .
$$

This shows that $\widehat{\psi}$ is also an eigenfunction corresponding to $\lambda_{2}$. Now put $\tilde{\psi}(x)=\psi(x)+\widehat{\psi}(x)$. Since $\psi$ is not antisymmetric with respect to the $x_{1^{-}}$ axis, $\tilde{\psi}$ is not identically zero. So $\tilde{\psi}$ is an eigenfunction corresponding to $\lambda_{2}$ which is symmetric with respect to the $x_{1}$-axis. Defining $\tilde{\tilde{\psi}}(x)=\tilde{\psi}(x)+\tilde{\psi}(\check{x})$ $\left(\check{x}=\left(x_{1},-x_{2}\right)\right.$, where $\left.x=\left(x_{1}, x_{2}\right)\right)$, the same argument shows that $\tilde{\tilde{\psi}}$ is not identically zero and that it is an eigenfunction corresponding to $\lambda_{2}$ which is symmetric with respect to both the $x_{1}$-axis and the $x_{2}$-axis. We may thus assume that the second eigenfunction $\varphi_{2}\left(\left\|\varphi_{2}\right\|_{2}=1\right)$ is symmetric with respect to both coordinate axes. By Lemma 4.2 we have

$$
\lambda_{2}-\lambda_{1} \geq e^{-2 \lambda_{1} T} \int_{0}^{T} \int_{D}\left|\nabla\left(\frac{u_{2}}{u_{1}}\right)(x, t)\right|^{2} \varphi_{1}^{2}(x) d x d t,
$$

where $T>0$ is arbitrary.

We will apply Lemma 4.5 to the integral on the right-hand side of (4.6) for $u(x, t)=u_{2}(x, t) / u_{1}(x, t)$. First, we need to check that the function $u(x, t)$ satisfies the hypothesis of the lemma. By Theorem 2.1 and Definition 2.3(i) in [6], $u$ is continuous and bounded on $D \times[0,1]$. 
We will show that for any fixed $x \in D, u(x, \cdot) \in C^{1}[0,1]$. We have

$$
\frac{\partial u}{\partial t}(x, t)=\frac{\frac{\partial u_{2}}{\partial t}(x, t) u_{1}(x, t)-\frac{\partial u_{1}}{\partial t}(x, t) u_{2}(x, t)}{u_{1}^{2}(x, t)} .
$$

Also, $u_{2}, u_{1}$ are bounded and continuous on $D \times[0,1]$ and $u_{1}$ is strictly positive on $D \times[0,1]$. We also have (formula (3.3) in [5])

$$
\frac{\partial u_{n}}{\partial t}(x, t)=-\lambda_{n} u_{n}(x, t)+T_{t} r_{n}(x), \quad x \in \mathbb{R}^{2}, \quad t>0,
$$

for all $n$ where $r_{n}$ is defined by formula (3.1) in [5]. $r_{n} \in L^{1}\left(\mathbb{R}^{2}\right)$ by Proposition 3.2(i) in [5]. It follows that $T_{t} r_{n}$ is continuous on $D \times(0,1]$. Since $r_{n} \in L^{1}\left(\mathbb{R}^{2}\right)$ and $\operatorname{supp}\left(r_{n}\right) \subset D^{c}$ (see Proposition 3.1 in [5]), it follows that $\lim _{t \rightarrow 0^{+}} T_{t} r_{n}(x)=0, x \in D$. Indeed, for $x \in D$

$$
\left|T_{t} r_{n}(x)\right|=\left|\int_{D} p(t, x, y) r_{n}(y) d y\right| \leq \frac{t}{2 \pi\left(t^{2}+\delta_{D}^{2}(x)\right)^{3 / 2}} \int_{D}\left|r_{n}(y)\right| d y,
$$

where $\delta_{D}(x)=\operatorname{dist}(x, \partial D)$. This gives $\lim _{t \rightarrow 0^{+}} T_{t} r_{n}(x)=0, x \in D$. It follows that for any fixed $x \in D, u(x, \cdot) \in C^{1}[0,1]$. By Theorem $2.1 \varphi_{1}$ is symmetric with respect to both axes and by our assumption $\varphi_{2}$ is also symmetric with respect to both axes. It follows that for any $i=1,2$ and any fixed $t \in(0,1] u_{i}(\cdot, t)$ is symmetric with respect to both axes on $D$ and of course, $u_{i}(\cdot, t) \in C^{1}(D)$ with

$$
\int_{D} u(x, 0) \varphi_{1}^{2}(x) d x=\int_{D} \frac{u_{2}(x, 0)}{u_{1}(x, 0)} \varphi_{1}^{2}(x) d x=\int_{D} \frac{\varphi_{2}(x)}{\varphi_{1}(x)} \varphi_{1}^{2}(x) d x=0 .
$$

Thus the function $u=u_{2} / u_{1}$ satisfies the assumptions of Lemma 4.5. Hence it follows that for any $T \in(0,1]$,

$$
\lambda_{2}-\lambda_{1} \geq \frac{C_{\varphi}}{L^{2}} e^{-2 \lambda_{1} T} \int_{0}^{T} \int_{D}\left(u^{2}(x, t)+\left(\frac{\partial u}{\partial t}(x, t)\right)^{2}\right) \varphi_{1}^{2}(x) d x d t
$$

where $C_{\varphi}$ is the same constant as in Lemma 4.3. By Lemma 4.6 the last quantity is bounded below by

$$
\begin{aligned}
\frac{C_{\varphi} T}{2 L^{2}} e^{-2 \lambda_{1} T} \int_{D} u^{2}(x, 0) \varphi_{1}^{2}(x) d x & =\frac{C_{\varphi} T}{2 L^{2}} e^{-2 \lambda_{1} T} \int_{D} \frac{\varphi_{2}^{2}(x)}{\varphi_{1}^{2}(x)} \varphi_{1}^{2}(x) d x \\
& =\frac{C_{\varphi} T}{2 L^{2}} e^{-2 \lambda_{1} T}
\end{aligned}
$$


where $T \in(0,1]$. On $[0, \infty)$ the function $T e^{-2 \lambda_{1} T}$ attains its maximum for $T=1 /\left(2 \lambda_{1}\right)$ and this maximum is $1 /\left(2 e \lambda_{1}\right)$. Note that the inradius $r_{D}$ of $D$ satisfies $1 \geq r_{D} \geq \sqrt{2} / 2$. By Corollary 2 in [8] we have $\lambda_{1} \geq 1$ so $1 /\left(2 \lambda_{1}\right) \in(0,1]$. Hence

$$
\lambda_{2}-\lambda_{1} \geq \frac{C_{\varphi}}{4 e \lambda_{1} L^{2}} .
$$

On the other hand, $\lambda_{1}=\lambda_{1}(D) \leq \lambda_{1}(B(0, \sqrt{2} / 2))=\sqrt{2} \lambda_{1}(B(0,1))$ (Here we write $\lambda_{1}(D), \lambda_{1}(B(0,1))$, etc., for the first eigenvalue $\lambda_{1}$ for the domains $D$ and $B(0,1)$.) By Corollary 2.2 (formula $(2.15)$ ) from $[5], \lambda_{1}(B(0,1)) \leq 2 \pi / 3$, so $\lambda_{1}=\lambda_{1}(D) \leq 2 \sqrt{2} \pi / 3$. With $C_{\varphi}=4 \cdot 10^{-6}$ it follows that

$$
\lambda_{2}-\lambda_{1} \geq \frac{12 \cdot 10^{-6}}{8 \sqrt{2} e \pi L^{2}} \geq \frac{10^{-7}}{L^{2}} .
$$

\section{References}

[1] M. S. Ashbaugh and R. Benguria, Optimal lower bounds for eigenvalue gaps for Schrödinger operators with symmetric single-well potentials and related results, Maximum principles and eigenvalue problems in partial differential equations, Longman, White Plains, NY, 1988.

[2] M. Ashbaugh and R. Benguria, Optimal lower bounds for the gap between the first two eigenvalues of one-dimensional Schrödinger operators with symmetric single-well potentials, Proc. Amer. Math. Soc., 105 (1989), 419-424.

[3] M. S. Ashbaugh and R. Benguria, A sharp bound for the ratio of the first two eigenvalues of Dirichlet Laplacians and extensions, Ann. Math. 135(2) (1992), 601-628.

[4] R. Bañuelos, Intrinsic ultracontractivity and eigenfunction estimates for Schrödinger operators, J. Funct. Anal. 100 (1991), 181-206.

[5] R. Bañuelos, T. Kulczycki, The Cauchy process and the Steklov problem, J. Funct. Anal. 211 (2004), 355-423.

[6] R. Bañuelos, T. Kulczycki, Eigenvalue gaps for the Cauchy process and a Poincaré inequality, preprint. 
[7] R. Bañuelos, T. Kulczycki, P. J. Méndez-Hernández, On the shape of the ground state eigenfunction for stable processes, To appear in Potential Analysis.

[8] R. Bañuelos, R. Latała, P. J. Méndez-Hernández, A Brascamp-LiebLuttinger-type inequality and applications to symmetric stable processes, Proc. Amer. Math. Soc. 129(10) (2001), 2997-3008 (electronic).

[9] R. Bañuelos, P. J. Méndez-Hernández, Sharp inequalities for heat kernels of Schrödinger operators and applications to spectral gaps, J. Funct. Anal. 176(2) (2000), 368-399.

[10] M. van den Berg, On condensation in the free-boson gas and the spectrum of the Laplacian, J. Statist. Phys., 31 (1983), 623-637.

[11] R.M. Blumenthal and R.K. Getoor, Markov Processes and Potential Theory, Springer, New York, 1968.

[12] R.M. Blumenthal and R.K. Getoor, The asymptotic distribution of the eigenvalues for a class of Markov operators Pacific J. Math. 9 (1959), 399-408.

[13] R.M. Blumenthal, R.K. Getoor, D.B. Ray On the distribution of first hits for the symmetric stable processes, Trans. Amer. Math. Soc. 99 (1961) 540-554.

[14] K. Bogdan and T. Byczkowski Potential theory for the $\alpha$-stable Schrödinger operator on bounded Lipschitz domains, Studia Math. 133(1) (1999), 53-92.

[15] K. Burdzy, T. Kulczycki Stable processes have thorns, Ann. Probab. 31(1) (2003), 170-194.

[16] Z.Q. Chen and R. Song, Intrinsic ultracontractivity and conditional gauge for symmetric stable processes, J. Funct. Anal. 150(1) (1997), 204-239.

[17] Z.Q. Chen and R. Song, Intrinsic ultracontractivity, conditional lifetimes and conditional gauge for symmetric stable processes on rough domains, Illinois J. Math. 44(1) (2000), 138-160.

[18] Z.Q. Chen and R. Song, Two sided eigenvalue estimates for subordinate Brownian motion in bounded domains, J. Funct. Anal. (to appear) 
[19] E.B. Davies, Heat Kernels and Spectral Theory, Cambridge University Press, Cambridge, 1989.

[20] B. Davis, On the spectral gap for fixed membranes, Ark. Mat. 39(1) (2001), 65-74.

[21] R. D. DeBlassie, Higher order PDEs and symmetric stable processes Probab. Theory Related Fields (2004).

[22] R. D. DeBlassie and P. J. Méndez-Hernández, $\alpha$-continuity properties of symmetric $\alpha$-stable process, Preprint.

[23] B. Dittmar, A. Y. Solynin, The mixed Steklov eigenvalue problem and new extremal properties of the Grötzsch ring. (Russian), Zap. Nauchn. Sem. S.-Peterburg. Otdel. Mat. Inst. Steklov. (POMI) 270 (2000),Issled. po Linein. Oper. i Teor. Funkts. 28, 51-79, 365.

[24] R. K. Getoor, Markov operators and their associated semi-groups, Pacific J. Math. 9 (1959) 449-472.

[25] J. Hersch, L. E. Payne, Extremal principles and isoperimetric inequalities for some mixed problems of Stekloff's type, Z. Angew. Math. Phys. 19 (1968), 802-817.

[26] N. Ikeda, S. Watanabe On some relations between the harmonic measure and the Levy measure for a certain class of Markov processes, J. Math. Kyoto Univ. 2 (1962), 79-95.

[27] T. Kulczycki, Intrinsic ultracontractivity for symmetric stable processes, Bull. Polish Acad. Sci. Math. 46(3) (1998), 325-334.

[28] R. Lavine, The eigenvalue gap for one dimensional convex potentials, Proc. Amer. Math. Soc., 121 (1994), 815-821.

[29] J. Ling, A lower bound for the gap between the first two eigenvalues of Schrödinger operators on convex domains in $S^{n}$ or $R^{n}$, Michigan Math. J. 40(2) (1993), 259-270.

[30] L. E. Payne and H. F. Weinenberg, An optimal Poincaré inequality for convex domains, Arch. Rational Mech. Anal. 5 (1960), 286-292.

[31] I. M. Singer, B. Wong, S.-T. Yau, S. S.-T. Yau, An estimate of the gap of the first two eigenvalues in the Schrödinger operator, Ann. Scuola Norm. Sup. Pisa Cl. Sci. (4) 12(2) (1985), 319-333. 
[32] R. G. Smits, Spectral gaps and rates to equilibrium for diffusions in convex domains Michigan Math. J. 43(1) (1996), 141-157.

[33] S.-T. Yau, Open problems in geometry, in "Chern-a great geometer of the twentieth century," Edited by S.-T. Yau, International Press, Hong Kong, 1992.

[34] Q. Yu and J. Q. Zhong, Lower bounds of the gap between the first and second eigenvalues of the Schrödinger operator, Trans. Amer. Math. Soc. 294 (1986), 341-349. 